\title{
<TITRE> Rapport à l'argent et conception de la corruption politique
}

\author{
Pierre Lascoumes, directeur de recherche CNRS, Centre d’études européennes, Sciences-po, \\ Paris \\ Viviane Le Hay, Ingénieur CNRS, Centre Emile Durkheim, Sciences-po, Bordeaux
}

\begin{abstract}
« La collusion de la presse, de la finance et de la politique a créé une grouillante faune où l'on trafique et agiote en Bourse comme on trafique et agiote en politique ", J. L. Bory, préface à Bel ami de G. de Maupassant, 1973, p.10
\end{abstract}

RESUME - Les relations entre l'argent et le politique ont toujours été problématiques. C’est toujours la crainte d'une emprise des intérêts privés sur l'intérêt public qui est en cause, c'està-dire le dévoiement du bien commun par les besoins particularistes. Sur la base d'une enquête quantitative et de traitements statistique cet article se propose de préciser dans quelle mesure les représentations de l'argent privé et des moyens d'enrichissement influent sur la conception de la fonction politique. La démonstration procède en quatre temps. Après avoir présenté les jugements généraux sur l'argent et l'enrichissement, nous montrerons leur relation avec l'appréciation du politique. Puis, nous traiterons des relations entre l'image de l'argent et les jugements sur la probité publique. Enfin, nous présenterons une typologie d’attitudes qui synthétise les variables précédentes.

$<$ TEXTE $>$ Les relations entre argent et politique ont toujours été problématiques et leurs rapports sont le plus souvent présentés comme antagonistes. L’activité politique est depuis les polémiques dans les cités grecques puis romaines régulièrement mise en cause sous l'angle des rapports intéressés entretenus par les détenteurs de l'autorité publique avec l'argent public et/ou avec les acteurs économiques. La figure du sycophante témoigne de l'ancienneté de cette préoccupation ${ }^{1}$ (Mélèze, 1987 ; Rivière, 2005 ; Doganis, 2007). En France, les grands classiques que sont les romans G. de Maupassant (Bel ami, 1884) et de E. Zola (L'argent, $1898^{2}$ ) décortiquent les effets délétères des rapports de connivence entre les milieux de la finance et de la politique. Simmel synthétise la question de la façon suivante ${ }^{3}$ : «Rien ne

\footnotetext{
${ }^{1}$ Il s’agissait à l'origine de citoyens vigilants dénonçant sous protection un dysfonctionnement dans la Cité. Ils exerçaient alors la fonction de Ministère public dans un système judiciaire qui en était dépourvu. Mais l'institution a été retournée contre elle-même et la dénonciation est devenue pour certaines personnes une source de revenus et l'objet de multiples manipulations individuelles ou politiques. Le Sycophante est devenu l'archétype du délateur ou du calomniateur doté d'intentions perverses.

${ }^{2}$ Au-delà de l’arrivisme du héros et de la collusion de la presse avec les puissances financières et politiques, Bel Ami transpose au Maroc «l'affaire tunisienne » qui a permis une vaste spéculation boursière. Le gouvernement et la Parlement y apparaissent comme totalement instrumentalisés par une guerre entre banques $-L$ 'argent est le $18^{\circ}$ volume de la série des Rougon-Macquart basé sur l'histoire de la faillite de la «Banque universelle ». Il présente la lutte à mort de deux banquiers (Saccard/Gundermann) sur un fond de manipulations politiques constantes. Dans la même veine : Anatole France, L'île des pingoins, 1908.

${ }^{3}$ Il donne de la notion une définition large : " La corruption : vendre son devoir ou sa conviction », mais les exemples qu'il donne sont pour l'essentiel politiques (Charles V, le parlement irlandais, p. 488-489) ou concernent l'Eglise catholique (p. 486)
} 
facilite et ne développe davantage les comportements corruptibles et la réalité de la corruption dans son ensemble que la forme argent de celle-ci. Foncièrement, l'argent favorise comme nulle autre valeur les changements de propriétés occultes, hors de vue et sans bruit. Comprimable, il permet de rendre quiconque riche, d'un simple morceau de papier glissé dans une main; amorphe et abstrait, il s'investit dans les valeurs les plus diverses, les plus lointaines, et se soustrait aux regards de l'entourage immédiat (...) Alors que, d'un côté, la faculté d'exprimer en argent toutes les valeurs fournit à l'acteur économique la vue la plus claire et la moins voilée sur l'état de ses biens, de l'autre elle permet de dissimuler devant autrui, jusqu'à l'inconnaissable, et la richesse des transactions, à un degré où les formes de la propriété extensive ne l’ont jamais admis » (Simmel, 1987, 485). Deux dimensions sont ainsi soulignées. D'une part, les rapports observables entre argent et politique ne sont qu'une déclinaison particulière des effets du développement d'une économie monétaire. D’autre part, l'argent autorise une des conditions essentielles des rapports corrompus : la dissimulation, propriété qui est contraire aux exigences de transparence démocratique ${ }^{4}$. Les traits relevés par Simmel sont une constante des écrits littéraires, d'essayistes ou de scientifiques sur le manque de probité dans les activités politiques et sur l'importance des pratiques intéressées menées sous couvert de la défense du bien commun. La « corruption » est alors entendue dans un sens extensif comme l'abus rémunéré d'une fonction publique. Elle s'applique aussi bien aux élus, aux ministres et aux fonctionnaires. Dans les relations entre l'argent et le politique c'est toujours la crainte d'une emprise des intérêts privés sur l'intérêt public qui est en cause, celle du dévoiement du bien commun par les besoins particularistes.

Durant ces 15 dernières années en France, l’opinion des citoyens considérant que « les élus sont plutôt corrompus » a progressé considérablement. Cet indicateur a surtout beaucoup augmenté dans les années 80 en relation avec les premières grandes affaires judiciaires de financement illicite des partis. Il passe alors de 38\% en 1977 à 55 \% en 1990. Il atteint 65\% en 1991 et se maintient depuis entre 60 et $65 \%{ }^{5}$ (Mayer, 2002 ; Lascoumes 2010). A l'occasion de notre enquête ${ }^{6}$ effectuée en 2006, un tiers des personnes enquêtées (32,6\%) estimait qu'il y a plus de corruption aujourd'hui qu'avant. Pour 61,6\% «il y a autant de

\footnotetext{
4 «Dans les conditions modernes, fortement composites, de la vie publique, avec ses mille énergies souterraines prêtes à rayonner partout, essentiellement liées à l’économie monétaire, la vénalité des fonctionnaires a des effets bien plus pernicieux » (Simmel, 1987, p. 490).

${ }^{5}$ La même question est posée par la SOFRES depuis 27 ans : « Diriez vous qu’en règle générale, les élus, les dirigeants politiques sont plutôt honnêtes ou plutôt corrompus ? ». Un niveau de réponse maximal a été à nouveau atteint à l'automne 2013 au moment de l'affaire Woerth-Bettancourt.

${ }^{6} \mathrm{Cf}$ présentation à la fin de l'introduction
} 
corruption aujourd'hui qu'avant mais on en parle davantage ». Seuls 4,4\% considèrent qu'il y en a moins. Cette évolution peut sembler paradoxale. En effet, jusqu’au milieu des années quatre-vingt aucune mesure spécifique n’encadrait le financement des activités politiques (fonctionnement des partis, élections, rémunération des élus) ${ }^{7}$. A partir de 1988 une quinzaine de lois et décrets ont été adoptés afin d’organiser un financement public et un certain degré de transparence des ressources et des dépenses ${ }^{8}$. Mais cette production de normes et sa mise en œuvre ont eu peu d'effets dans le rétablissement de la confiance des citoyens à l'égard de la probité de leurs dirigeants.

Les travaux d'historiens ont été les premiers en France à analyser les rapports de collusion entre les grandes entreprises, les banques et les acteurs politiques (ministres et députés) (Jeanneney, 1976, 1981). L'étude de plusieurs grands procès de la III ${ }^{\circ}$ République a fourni beaucoup d’éléments dans ce sens (Ferry, 1868 ; Desanti, 1968 ; Mollier, 1991). La sociologie des scandales politiques et financiers qui analyse la production et les traitements différentiels des « affaires » revient en permanence sur la perméabilité des décideurs publics aux intérêts privés (Lascoumes, 1997 ; Garrigues, 2004 ; de Blic, 2005). En science politique les relations problématiques entre l'argent et la politique sont abordées sous deux angles principaux ${ }^{9}$. Un premier ensemble de travaux traite de la défiance croissante dans les démocraties (disaffected democraties), c'est-à-dire de la perte de confiance des citoyens à l'égard du système politique, de ses acteurs et de ses institutions. Ce phénomène est analysé de longue date au USA (Steffens, 1904 ; Heidenheimer, 1970 ; Pharr et Putnam, 2000) et maintenant en Europe (Mény, 1992 ; Della Porta, 1995 ; Rosanvallon, 2006 ; Algan, Cahuc, 2007 ; Algan, Cahuc, Zylbergerg 2012). Cette défiance est très souvent mise en relation avec : d'une part, une représentation négative des dirigeants considérés comme potentiellement corruptibles (surtout les ministres et les élus nationaux) ; et d’autre part, une incapacité des démocraties à prévenir et sanctionner efficacement ces abus de pouvoir. Un deuxième ensemble de travaux met

\footnotetext{
${ }^{7}$ Contrairement à une idée répandue, cela ne voulait pas dire que tout était permis pour autant. Le code pénal prévoyait un ensemble d'infractions d'atteinte à la probité publique (Livre IV section 3, articles 432-10 à 43217) dont la corruption passive et active, le trafic d'influence, la prise illégales d'intérêts, le détournement de fonds publics, etc..

${ }^{8}$ Commission pour la transparence financière de la vie politique (CTFVP, 1988), Commission nationale des comptes de campagne et des financements politiques (CNCCFP, 1990), Service central de prévention de la corruption (SCPC, 1993).

${ }^{9}$ Nous ne retenons pas ici volontairement les nombreux travaux sur les formes de la corruption politique, les facteurs qui la facilitent et leurs évolutions (Bayley, 1966 ; Heidenheimer 1989 ; Neild 2002 ; Lambsdorff 2007) - Nous écartons aussi les travaux qui abordent la corruption sous l'angle des échanges de services, de faveurs et du clientélisme car la dimension financière n’y est pas centrale (Padioleau 1975, Médard, 1976, Briquet-Sawicki 1998).
} 
l'accent sur les effets négatifs de la professionnalisation politique dans les représentations collectives de ce «métier inavouable » (Damamme, 1999 ; Phélippeau, 2002). Les analyses développées aussi par P. Bacot (1999) et D. Gaxie (2001) ont montré que les perceptions ordinaires dominantes des acteurs politiques reposent, d'une part sur les caractéristiques sociales de «privilégié » des élus (leur capacité à accéder à des fonctions spécifiques) et d'autre part, sur les opportunités que la charge leur permet de saisir afin de « tirer profit » de leur mandat. Le cumul de rétributions financières et symboliques renforce les jugements négatifs sur l'activité politique considérée alors comme un moyen d'enrichissement. Une autre enquête a montré l'importance du lien établi par les personnes interrogées entre l'existence d'un intérêt spécifique du personnel politique (la «défense du poste ») et la critique de leur supposée «malhonnêteté » (Norris, 1999). Si l'on sait depuis les travaux de Campbell et al. (1960) dans The American voter qu'à défaut d'argumentaires plus développés, les formes ordinaires de critique de la politique reposent souvent sur des catégories morales quotidiennes (réprobation du mensonge, du carriérisme et de la cupidité), les travaux de D. Gaxie (2001) ont aussi montré que ce type de raisonnement s'observe aujourd'hui également dans les catégories sociales au capital socio-culturel plus élevé (classe moyenne, cadres).

L'objectif de cet article est d'investiguer sous un angle différent les relations entre argent et politique en mettant en relation les conceptions de l'argent privé et celles relatives à l'argent dans les activités politiques. Plus précisément nous chercherons à vérifier s'il existe (ou non) une relation entre d'un côté l'image individuelle de l'argent et des moyens de s'enrichir, et d'un autre côté les représentations de la dimension économique des activités politiques (rémunération, usage de l'argent public, probité). Nous faisons l'hypothèse qu'une image positive de l'argent et de l'enrichissement privé est reliée à une acceptation plus grande du rôle de l'argent dans les activités politiques. Inversement, une image négative de l'argent et de l'enrichissement privé devrait s'accompagner d'une réprobation plus marquée de l'argent dans les activités politiques.

Nous ferons une seconde hypothèse dans la suite des travaux sur le statut social de l'argent présenté dans l'introduction générale de ce numéro. La force du rationalisme économique croissant depuis quatre siècles, et la financiarisation quasi générale observable depuis une quarantaine d'années de toutes les activités sociales devraient avoir disqualifié toutes les attitudes ou rendu très marginales les positions hostiles à l'accumulation et à la circulation des 
richesses dites «anti-chrématistes» ${ }^{10}$ L'aboutissement de la logique rationnelle et pragmatique du capitalisme moderne dégagée par W. Sombart (1902) et M. Weber (1904) devrait déboucher au début du XXI ${ }^{\circ}$ siècle sur une émancipation complète de la conception de l'économie légitime à l'égard des normes religieuses. Telle que décrite par $\mathrm{M}$. Weber, la « conduite de vie chrématiste ", favorable à la monétarisation et aux échanges marchands, devrait donc être le modèle largement dominant dans les sociétés occidentales contemporaines. Il devrait aussi se traduire par une appréciation positive et pragmatique de la dimension financière des activités publiques. La frontière du modèle étant la figure du politicien corrompu, aux conduites égoïstement intéressées. Etant dans « la jouissance constante de sa puissance » (p.52) il incarnerait le modèle négatif de l’entrepreneur rationnel et ascétique. Et à ce titre il devrait être fortement rejeté. Qu'en est-il aujourd’hui de ce schéma théorique?

Les données empiriques utilisées proviennent d’une enquête menée au CEVIPOF sur les représentations sociales de la probité publique. L’enquête a combiné des terrains qualitatifs (monographies de trois municipalités, discussions de 11 focus group) et une enquête quantitative. Cette dernière a été réalisée en janvier 2006 par l’institut ISL, administrée en face à face au domicile des personnes interrogées (CAPI). Un échantillon de 2028 personnes a été constitué, représentatif de la population française âgée de 18 ans et plus. La représentativité de l'échantillon a été assurée par la méthode des quotas (sexe croisé par l’âge, profession ou dernière profession de la personne de référence du ménage, diplôme), après stratification par grandes régions (ZEAT) et catégories d'agglomération. La durée moyenne de passation du questionnaire est de 30 à 40 minutes.

Afin de montrer sous quelle forme les représentations de l'argent et des moyens d'enrichissement participent de la conception de la fonction politique, nous procèderons en quatre temps en présentant successivement :

- les jugements généraux sur l’argent privé

- les relations entre image de l’argent et les jugements généraux sur la politique

- les relations entre image de l’argent et les jugements sur la probité publique

- enfin une typologie d’attitudes qui synthétise les variables précédentes.

${ }^{10}$ La distinction a été élaborée par Aristote qui oppose les activités liées à l’oikos (répondant aux besoins humains) et les activités chrématistiques (contre nature, visant l’accumulation et basées sur l'échange mercantile).Vọir l’introduction générale de ce numéro.
Supprimé : (p. 436)

Supprimé : et à ce titre se retrouver dans

Supprimé : moins morale et plus

Supprimé : Sur 1

Supprimé : es notions de " chrématisme » et d' " antichrématisme "

Supprimé : v 


\section{$\leq \underline{\text { IT1 }>1 \text { 1. Rapports à l'argent et à l'enrichissement }}$}

<IT2> 1 - Jugements généraux sur l'argent

Dans un premier temps nous avons cherché à évaluer l'image générale de l'argent dans la population investiguée et cela de façon comparative. Il s'agissait de mesurer quelle valeur était attribuée à l'argent par rapport à d'autres notions ${ }^{11}$. Dans le prolongement des travaux présentés dans l'introduction, nous voulions cerner les positions à l'égard de plusieurs termes se référant à un ensemble de valeurs sociales et économiques de notre société, leur degré de cohérence ou d'ambivalence. Contrairement aux perceptions ordinaires qui présentent la financiarisation des activités humaines comme une caractéristique majeure de la société contemporaine, nous montrerons qu'une partie non-négligeable de la population enquêtée ne considère pas cette emprise économique sous un jour positif. La monétarisation n’a pas été naturalisée. Selon notre enquête aux environs du tiers des personnes interrogées se montrent au contraire critiques vis-à-vis de la place donnée à l'argent dans les rapports sociaux. Nous verrons que le vieux fond d'anti-chrématisme, de méfiance, voire de défiance à l'égard des activités économiques et des pratiques monétaires reste vivace.

L'image qui ressort vis-à-vis de l'argent est globalement positive : un peu plus des trois quarts de l'échantillon (77\%) partagent une perception positive, dont plus d'un quart (29\%) très positive. Les termes honnêteté et liberté d'expression sont ceux qui suscitent les jugements les plus positifs (très et assez positifs au-dessus de 88\%). L’Etat et le libéralisme économique suscitent à l'inverse les jugements les plus négatifs (plutôt et très négatifs au-dessus de 40\%). La perception de l'argent se situe en position médiane, au même niveau que l'autorité par exemple. Si on la compare avec la batterie de termes qui était proposée dans l'enquête, l'argent n'arrive qu'en 9e position dans les jugements très et assez positifs. Des termes comme honnêteté (90\%), liberté d'expression (89\%), solidarité (88\%), ambition (88\%), tolérance (88\%), travail (87\%), entreprise (83\%) et égalité (83\%) obtiennent de meilleurs scores.

\footnotetext{
${ }^{11}$ La question posée était : « Je vais vous citer une liste de mots. Pour chacun d'eux vous me direz s’ils évoquent pour vous quelque chose de très positif, assez positif, plutôt négatif, très négatif ? ». Successivement : argent travail, égalité, ambition, liberté d'expression, Etat, solidarité, libéralisme économique, honnêteté, autorité, entreprise. De manière générale, les Sans réponse ne représentent que de 0,1 à $0,8 \%$, ce qui indique que cette batterie de questions n’a pas posé de problème aux personnes interrogées. Seuls les termes «libéralisme économique » et « tolérance »ont recueilli une part plus importante de sans-réponse (resp. 6\% et 3\%).
} 
Les résultats de l'enquête European values study (EVS) de 2008 montrent une défiance plus nette à l'égard des valeurs monétaires. La question posée était différente mais proche dans sa signification : " Qu'on attache moins d'importance à l'argent et aux biens matériels, pensezvous que ce serait : une bonne chose, une mauvais chose, ou cela vous est-il égal ? ${ }^{12}$. Dans l'ensemble de l'enquête, le jugement «bonne chose » (moindre importance apportée à l'argent et aux biens matériels) atteint $67 \%$. En France, les réponses sont légèrement inférieures $(65 \%)^{13}$ et situent le pays dans la zone intermédiaire ${ }^{14}$ : les enquêtés de 15 pays se montrent plus défiants (niveau maximal pour Malte, Chypre et la Finlande (plus de $80 \%$ ) et ceux de 9 pays adhèrent davantage à ces valeurs (niveau maximal pour l'Autriche et la Lituanie 49\%).

Dans notre enquête, les jugements positifs relatifs à l'argent sont socialement largement répartis, tendanciellement ils sont plus fréquents chez les plus jeunes (moins de 24 ans), les personnes aux revenus supérieurs, en particulier chez les travailleurs indépendants, les agriculteurs et les inactifs (non retraités) et les électeurs de droite (UMP) et d'extrême droite (FN). Mais les écarts à la moyenne sont le plus souvent de faible ampleur. L'image est un peu plus négative chez les adultes âgés de 25 à 49 ans, les personnes aux revenus inférieurs $(<1500 €)$, les personnes travaillant dans la fonction publique nationale et territoriale et pour ceux qui déclarent une proximité politique de gauche (Verts, PC et extrême gauche). Le tableau en annexe 1 précise, pour un ensemble de ces variables, les tendances observées.

Par ailleurs, il faut souligner que les jugements portés sur l'argent sont très fortement liés à la représentation que les personnes interrogées se font du libéralisme économique et de l'entreprise. Si l'un est jugé négativement, alors les deux autres items ont toutes les chances de l'être à leur tour ${ }^{15}$.

\section{$\leq \underline{\mathrm{IT} 2}>2$ - Moyens légitimes et moins légitimes de s’enrichir}

\footnotetext{
${ }^{12}$ Enquête effectuée dans 28 pays européens auprès de 40773 personnes dont 1484 Français.

${ }^{13}$ Attacher moins d'importance à l'argent et aux biens matériels est : une bonne chose 65,4\%, une mauvaise chose $13,7 \%$, indifférent 20,9\%.

${ }^{14} \mathrm{Au}$ même niveau que la Belgique (65,8\%) et la Bulgarie (65,5\%).

${ }^{15}$ Cela a d'ailleurs permis de construire avec Guy Michelat une échelle d'attitude de type Loevinger à partir de ces trois variables.
} 
Une série de questions complétait la précédente en investiguant les jugements portés sur les moyens légitimes et moins légitimes ou illégitimes de gagner des sommes d'argent importantes ${ }^{16}$.

- Quatre moyens légitimes étaient proposés : « hériter », « épargner », « créer son entreprise », «faire des heures supplémentaires». Les moyens les plus approuvés sont «créer son entreprise » (Tout à fait normal et plutôt normal : 97\%), « épargner » et « hériter » (Tout à fait normal et plutôt normal : 93\%). « Faire des heures supplémentaires » est considéré normal par 79\% de l'échantillon.

- Quatre moyens moins légitimes voire illégitimes étaient également soumis à l'appréciation des répondants : «frauder le fisc », « cumuler des emplois », « employer des gens au noir » et « utiliser des combines ». Les moyens les plus réprouvés, et ce de manière presque unanime, sont « frauder le fisc ou la sécurité sociale » (plutôt pas normal et pas normal du tout : 96,5\%) et « employer des gens au noir » (plutôt pas normal et pas normal du tout : 92\%), tandis que 82\% de l'échantillon n'estiment pas normal d'utiliser des combines.

- «Cumuler des emplois » apparaît être le moyen le plus controversé : 51\% le jugent tout à fait normal et plutôt normal et $48 \%$ plutôt pas normal et pas normal du tout.

Ces premiers résultats méritent d'être approfondis. Nous allons essayer de comprendre plus globalement les lignes de clivages présentes au sein de l'échantillon et d'identifier les raisons pour lesquelles les répondants se positionnent (les uns par rapport aux autres) de manière plus ou moins hostile ou bienveillante vis-à-vis des façons de gagner de l'argent. Pour ce faire, nous avons construit deux indicateurs d'approbation des moyens légitimes de s'enrichir d'une part, et de ceux moins légitimes d'autre part. La démarche est statistiquement valide, dans la mesure où ces questions font échelle entre elles (les 4 questions relatives aux moyens légitimes obtiennent un alpha de Cronbach s'élevant à 0,597; cet indice atteint 0,650 pour ce qui concerne les moyens les moins légitimes).

Par ce biais, nous pouvons isoler d'un côté les 53\% de l'échantillon qui se montrent les plus favorables aux moyens légitimes de gagner de l'argent (approbation 53\% / réprobation 47\%), et d'un autre côté les $65 \%$ qui réprouvent clairement les moyens plus illégitimes de s'enrichir (réprobation 65\% / approbation 35\%).

Ainsi, dans la population étudiée, le dissensus est élevé sur la légitimité des formes d'enrichissement. On observe deux clivages nets, aussi bien sur l'approbation des moyens

\footnotetext{
${ }^{16}$ La question était : « Trouvez vous normal de gagner beaucoup d’argent en ... » (liste de moyens). Les réponses possibles étaient : tout à fait normal, plutôt normal, plutôt pas normal, pas normal du tout.
} 
légitimes (53\%/47\%) que sur la réprobation des moyens illégitimes (65\%/35\%). Ces résultats permettent d'identifier une première forme d'ambivalence à l'égard de l'argent et des moyens d'en obtenir.

Quelles sont les spécificités de chacun de ces sous-groupes (cf tableau en annexe 1)?

Les personnes qui approuvent le plus les moyens légitimes sont surtout âgées de 18 à 49 ans, de qualification moyenne (primaire supérieur et Bac), les travailleurs indépendants, les personnes déclarant une appartenance religieuse non catholique et une proximité partisane de droite (UMP, FN).

Les personnes qui approuvent le plus les moyens illégitimes sont surtout les jeunes (18-24 ans), de qualification supérieure (Bac et + ), les chefs d'entreprise et professions libérales ${ }^{17}$, mais aussi les ouvriers et les inactifs, les personnes déclarant une appartenance religieuse non catholique et une proximité partisane avec les partis périphériques (extrême gauche, Verts et FN).

\section{$\leq$ IT2 $>3$ - Attitudes chrématiste et anti-chrématiste}

Le résultat le plus intéressant est le suivant. De façon contre-intuitive le clivage principal dans notre population ne porte pas sur l'opposition attendue entre ceux qui n'adhèrent qu'aux moyens légitimes et ceux qui adhèrent à tous les moyens qu'ils soient légitimes ou illégitimes. Nous observons une séparation différente qui repose non pas sur le choix des moyens, mais sur le principe même de l'enrichissement. En effet, deux groupes bien contrastés sur cet enjeu représentent plus de la moitié de notre échantillon (55\%). D’un côté se trouvent ceux qui refusent le principe même de l'enrichissement et qui donc refusent tous les moyens y compris ceux qui sont légitimes (leur position est ainsi typiquement antichrématiste, $n=33 \%$ ). Le tableau $n^{\circ} 1$ montre la relation entre les refus des moyens légitimes (rejet de deux moyens et plus) et les refus de moyens illégitimes (rejet de trois moyens et plus). D'un autre côté, se trouve le groupe de ceux qui au contraire valorisent l'enrichissement et adhèrent à tous les moyens d'y parvenir, même ceux qui sont illégitimes (leur position est typiquement pro-chrématiste, $\mathrm{n}=22 \%$ ). Le même tableau montre l'existence d'une relation symétrique entre l'acceptation des moyens légitimes (trois moyens et plus) et l'acceptation des moyens illégitimes (trois moyens et plus).

\footnotetext{
${ }^{17}$ Ce résultat qui peut paraître contre-intuitif (la conception fonctionnaliste de la dévance met en relation l'insertion sociale - l'accès aux opportunités légitimes - et le respect des normes), mais il rejoint une étude de psycho-sociologie récente qui montre sur une base empirique une corrélation entre statut social favorisé et capacité à enfreindre les règles. Les auteurs interprètent cela comme une « perception plus favorable de la cupidité » dans les classes supérieures (Piff, Stancato and al. 2012)
} 


\begin{tabular}{|c|c|c|c|c|c|c|}
\hline & & \multicolumn{5}{|c|}{ Nombre de moyens illégitimes considérés normaux } \\
\hline \multirow{6}{*}{$\begin{array}{c}\text { Nombre } \\
\text { de moyens } \\
\text { légitimes } \\
\text { considérés } \\
\text { tout à fait } \\
\text { normaux }\end{array}$} & & 0 sur 4 & 1 sur 4 & 2 sur 4 & $3-4$ sur 4 & Total \\
\hline & 0-1 sur 4 & 42 & 31 & 16 & 11 & 100 \\
\hline & 2 sur 4 & 32 & 35 & 18 & 15 & 100 \\
\hline & 3 sur 4 & 31 & 31 & 20 & 18 & 100 \\
\hline & 4 sur 4 & 17 & 39 & 20 & 23 & 100 \\
\hline & Total & 31 & 34 & 19 & 17 & 100 \\
\hline
\end{tabular}

Tableau $\mathrm{n}^{\circ} 1$ - Combinaison des jugements à l’égard des moyens légitimes et illégitimes de s’enrichir

Le tableau $n^{\circ} 2$ précise en pourcentage les deux groupes présentés ci-dessus (groupe 1 antichrématiste 33\% et le groupe 2 pro-chrématiste 22\%) et les deux groupes intermédiaires. Le groupe $n^{\circ} 3$ (31\%) concerne de façon attendue les enquêtés qui adhèrent aux moyens légitimes (trois ou quatre moyens) et désapprouvent les moyens illégitimes (deux et plus). On peut être étonné que ce groupe ayant le profil normatif le plus prévisible ne représente que le tiers des répondants. A l'inverse, le groupe $n^{\circ} 4$ est le plus restreint. Il peut être considéré comme celui des déviants dans la mesure où de façon paradoxale ses membres (14\%) adhèrent aux moyens illégitimes (trois ou quatre moyens) tout en refusant en partie les moyens légitimes (trois et plus)

\begin{tabular}{|c|c|c|c|c|c|}
\hline & & \multicolumn{4}{|c|}{ Nombre de moyens illégitimes considérés normaux } \\
\hline \multirow{5}{*}{$\begin{array}{c}\text { Nombre de } \\
\text { moyens } \\
\text { légitimes } \\
\text { considérés } \\
\text { tout à fait } \\
\text { normaux }\end{array}$} & & 0 sur 4 & 1 sur 4 & $2 \operatorname{sur} 4$ & $3-4$ sur 4 \\
\hline & $0-1$ sur 4 & \multirow{2}{*}{\multicolumn{2}{|c|}{$\begin{array}{l}\text { Groupe } 1 \text { : Les anti- } \\
\text { chrématistes (33\%) }\end{array}$}} & \multirow{2}{*}{\multicolumn{2}{|c|}{$\begin{array}{c}\text { Groupe } 4 \text { : intermédiaire } \\
\qquad(14 \%)\end{array}$}} \\
\hline & $2 \operatorname{sur} 4$ & & & & \\
\hline & $3 \operatorname{sur} 4$ & \multirow{2}{*}{\multicolumn{2}{|c|}{$\begin{array}{c}\text { Groupe } 3 \text { : intermédiaire } \\
(31 \%)\end{array}$}} & \multirow{2}{*}{\multicolumn{2}{|c|}{$\begin{array}{l}\text { Groupe } 2 \text { : les pro- } \\
\text { chrématistes (22\%) }\end{array}$}} \\
\hline & $4 \operatorname{sur} 4$ & & & & \\
\hline
\end{tabular}

Tableau 2 - Les quatre groupes d'attitudes à l'égard des moyens d'enrichissement

Si l'on croise cette typologie avec l'image générale de l'argent des membres de chacun de ces groupes la distinction est confirmée pour l'essentiel (tableau ${ }^{\circ} 3$ ). C'est le groupe antichrématiste qui a l'image la moins favorable de l'argent (la moyenne de l'ensemble de notre population est de $29 \%$ pour les «très positifs » et de $48 \%$ pour les «assez positifs » (cf supra).

\begin{tabular}{|l|c|c|c|c|}
\hline & Très positif & Assez positif & Plutôt négatif & Très négatif \\
\hline Pro-chrématistes & $\mathbf{3 7}$ & 42 & 15 & 6 \\
\hline
\end{tabular}




\begin{tabular}{|l|l|l|l|l|}
\hline Anti-chrématistes & $\mathbf{2 1}$ & 52 & 22 & 5 \\
\hline Groupes intermédiaires & 30 & 48 & 17 & 5 \\
\hline
\end{tabular}

Tableau 3 - Attitude pro/anti-chrématistique et image de l’argent

Enfin, nous avons caractérisé le profil socio-économique de chacun de ces groupes (Tableau en annexe 1):

- Groupe 1: Les anti-chrématistes (33\%) sont surtout des adultes et des personnes âgées (50 ans et plus), faiblement diplômées (sans diplôme et primaire). Les salariés de l'Etat (en particulier enseignants), retraités et inactifs sont fortement représentés. Il en va de même des catholiques pratiquants. Leur positionnement politique est en partie de gauche (surtout le parti communiste et plus faiblement le PS) ou bien l'apolitisme (aucun parti).

- Groupe 2 : Les pro-chrématistes (22\%) sont surtout des jeunes et jeunes adultes (18-34 ans), diplômés (bac et +). Il s'agit souvent de salariés du secteur privé et de personnes ayant des revenus supérieurs à $3000 €$. Ceux qui se déclarent d'une religion autre que le catholicisme sont plus nombreux. Leur positionnement politique est plutôt de droite (avec une proximité avec le FN), mais on trouve aussi des personnes sans attachement partisan déclaré et d'autres se déclarant proche des Verts.

\section{$\leq$ IT1 $>2$. Argent et conceptions du politique}

$<$ IT2 $>1$. Perception de l'argent et Perception de la politique

Dans le prolongement des travaux présentés en introduction ayant montré l'importance des questions d'argent dans les images négatives et dans la défiance à l'égard des acteurs et de la vie politique en général, nous avons investigué de plusieurs façons cet enjeu. Les réponses à trois questions nous ont permis de cerner les liens qui peuvent exister entre les perceptions de l'argent et celles de la confiance dans les acteurs politiques, de l'honorabilité de la fonction et de la professionnalisation du métier ${ }^{18}$.

\footnotetext{
${ }^{18}$ Les résultats généraux de l'enquête donnent - Confiance a : augmenté 3\%, restée la même 42\%, diminué 55\%, NSP 1\% - Honorabilité de l'activité : Oui 67\%, Non 30\%, NSP 3\% - Politique comme métier : Plutôt une bonne chose, $47 \%$, Plutôt une mauvaise chose $48 \%$, NSP 5\%.
} 


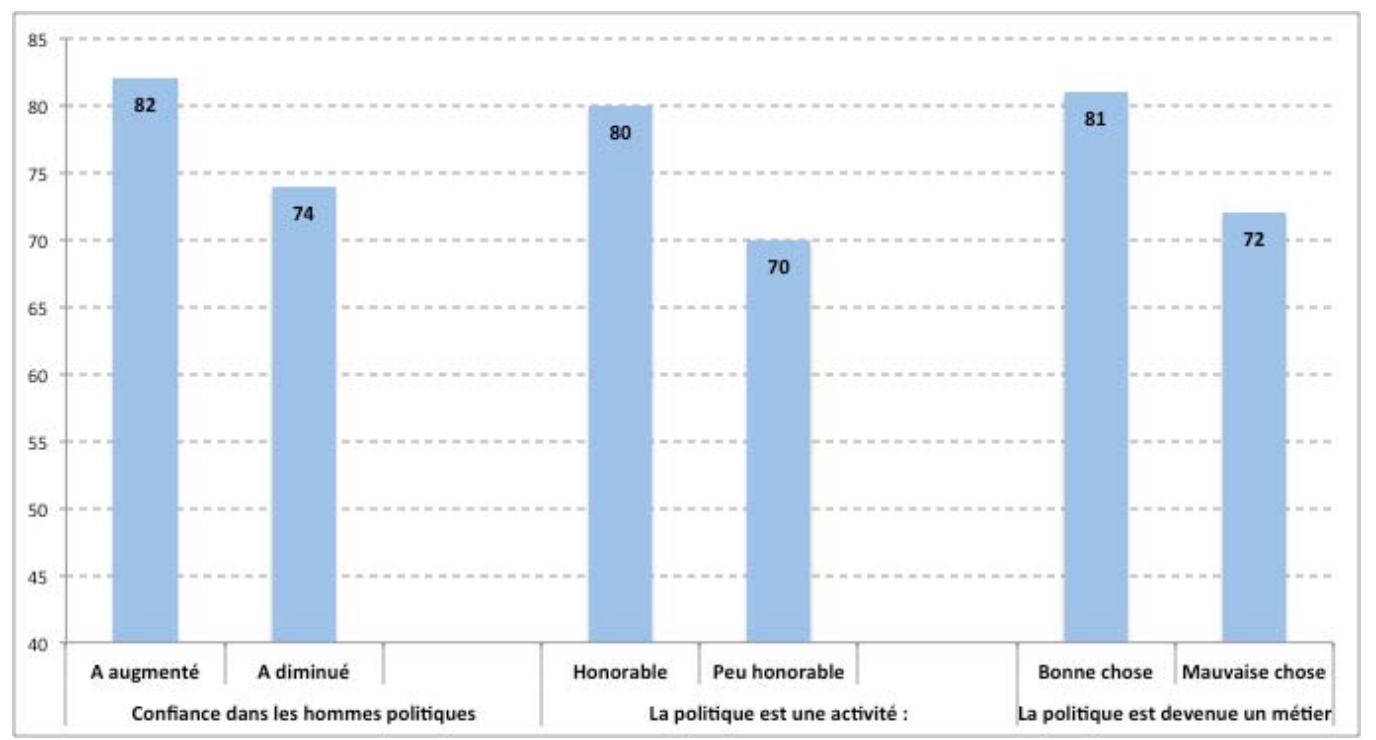

Figure $n^{\circ} \mathrm{I}$ - Personnes ayant une perception positive de l'argent* et leur confiance dans les hommes politiques, leur jugement sur l'honorabilité de l'activité et leur perception de la politique comme métier.

* Par souci de lisibilité apparaissent ici uniquement le pourcentage des personnes ayant une image positive de l'argent. Les personnes ayant une image négative sont le complément à $100 \%$ de ce qui apparaît sur la figure.Ex: $18 \%$ des personnes estimant que leur confiance dans les hommes politiques a augmenté ont une image négative de l'argent $(100-82=18)$

Il existe un lien statistique significatif ${ }^{19}$ entre la perception de l'argent et les trois variables rendant compte d'un rapport plus ou moins distancié et défiant à la politique, validant l'hypothèse d'un rapport étroit entre image positive de l'argent et meilleure perception de l'activité politique (figure ${ }^{\circ} \mathrm{I}$ ).

Les personnes dont la confiance dans le personnel politique a augmenté, qui jugent la fonction plutôt honorable et qui approuvent la professionnalisation évaluent plus souvent de façon positive l’argent que celles qui témoignent une plus grande défiance sur ces trois dimensions (respectivement +8 points, +10 points et +9 points). A l'inverse donc, les personnes dont la confiance dans le personnel politique a diminué, qui jugent la fonction peu honorable et qui désapprouvent la professionnalisation sont aussi celles qui ont plus fréquemment que les autres une image négative de l'argent.

La construction d'un indicateur de confiance dans les acteurs politiques renforce ce constat. Les personnes qui se défient le plus des politiques (confiance diminuée, faible honorabilité, métier mauvais chose $=0$ attribut sur l'indicateur) ne sont que $67 \%$ à avoir une image positive de l'argent (tableau nº 4 ). Elles sont en revanche $88 \%$ dès lors qu'elles jugent positivement les

\footnotetext{
${ }^{19}$ Les tests du khi-deux sont significatifs.
} 
acteurs politiques à trois reprises ( 3 attributs $=+21$ points). Nous observons ici une logique cumulative selon laquelle, plus la confiance à l'égard du personnel politique est forte, plus la perception de l'argent est positive.

\begin{tabular}{|l|c|c|}
\hline & \multicolumn{2}{|c|}{ Image de l'argent } \\
\hline $\begin{array}{l}\text { Attributs de confiance vis- } \\
\text { à-vis du personnel politique }\end{array}$ & Positive & Négative \\
\hline Aucun & 67 & 33 \\
\hline 1 & 76 & 24 \\
\hline 2 & 80 & 20 \\
\hline 3 & 88 & 12 \\
\hline
\end{tabular}

Tableau n $^{\circ} 4$ - confiance dans les acteurs politiques (de 0 à 3 attributs) et image de l'argent

\section{$\leq \mathrm{IT} 2>2$. Rémunération des députés.}

Une autre question porte sur l'appréciation du niveau de rémunération des députés. C'est un point très sensible dans la mesure où le fait de «vivre de la politique » et la continuité des carrières que cela implique relève typiquement des stéréotypes négatifs à l'égard de l’activité politique (Figure $\left.{ }^{\circ} \mathrm{II}\right)$.

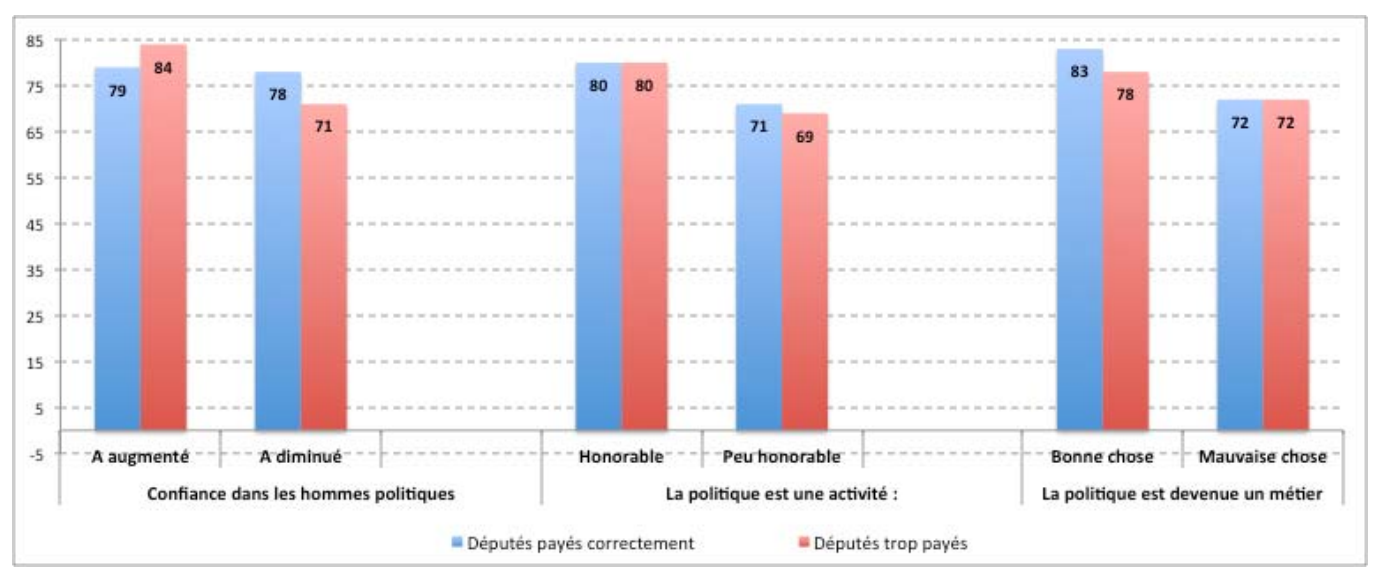

Figure $n^{\circ}$ II : Répartition des personnes ayant une image positive de l'argent selon leur opinion sur la rémunération des députés et leurs attitudes à l'égard du politique

Les variations sont un peu plus importantes pour les personnes estimant que les députés sont trop payés (50\% de la population enquêtée). Dans l'ensemble, elles sont de moindre ampleur pour ceux qui estiment que les députés sont payés correctement $(43 \%)^{20}$ et cela quelle que soit leur image de l'argent.

\footnotetext{
${ }^{20}$ Trop payés $50 \%$; Assez bien payés $29 \%$; Payés juste comme il faut $14 \%$; Pas assez payé 1\%, NSP 6\%.
} 
Les personnes qui considèrent que les députés sont trop payés ont une image plus négative de l'argent que celles qui estiment qu'ils sont payés correctement. En d'autres termes considérer que les députés sont trop payés va de pair avec la logique antichrématiste. On peut également y voir un ressentiment, un possible « jalousie sociale » à l'égard des élites politiques. L'effet de ces jugements sur le salaire des députés (correctement/trop payés) explique que l’on passe de $84 \%$ à $71 \%$ d'image positive de l'argent en fonction de sa confiance dans les hommes politiques, de $80 \%$ à $69 \%$ en fonction de l'honorabilité de l'activité politique et de $78 \%$ à $72 \%$ en fonction de la constatation qu'elle est devenue ou non un métier.

La figure $n^{\circ} \mathrm{III}$ confirme bien cette tendance : à mesure que la confiance dans les acteurs politiques (sous forme de l'indicateur de confiance, cf supra) s'accroît, l'image de l'argent est plus fréquemment positive. En revanche, le fait d'estimer que les députés sont trop payés, s'accompagne d'une image de l'argent légèrement revue à la baisse. Cela est d'ailleurs d'autant plus vrai que la confiance dans les acteurs politiques est faible.

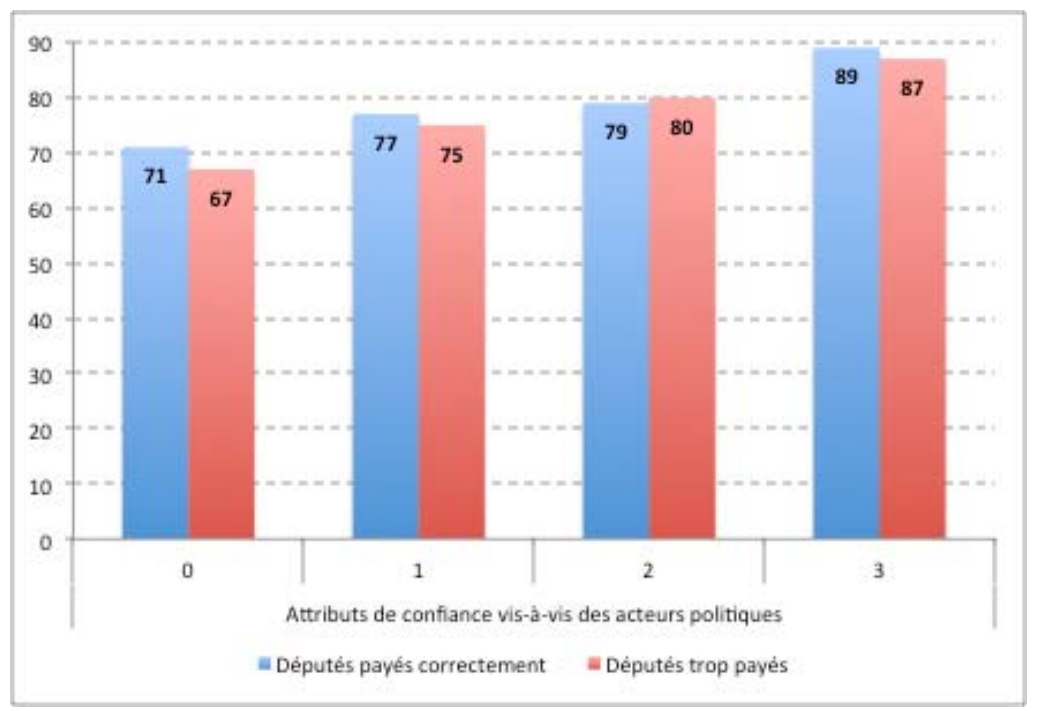

Figure $n^{\circ}$ III- Répartition des personnes ayant une image positive de l'argent selon leur opinion sur la rémunération des députés et leur attribut de confiance à l'égard du politique

\section{$\leq \mathrm{IT} 2>3$. Utilisation de l'argent public}

Une troisième façon d'envisager la question des relations entre argent et politique est de s'interroger sur la façon dont est perçue l'utilisation de l'argent public. Les résultats généraux de l'enquête donnent les proportions suivantes : Très bien utilisé 7\% ; Bien utilisé 54\% ; 
Plutôt mal utilisé $28 \%$; Très mal utilisé $10 \%$; NSP 1\% ; Très bien et Bien $61 \%$; Plutôt mal et très mal $38 \%$.

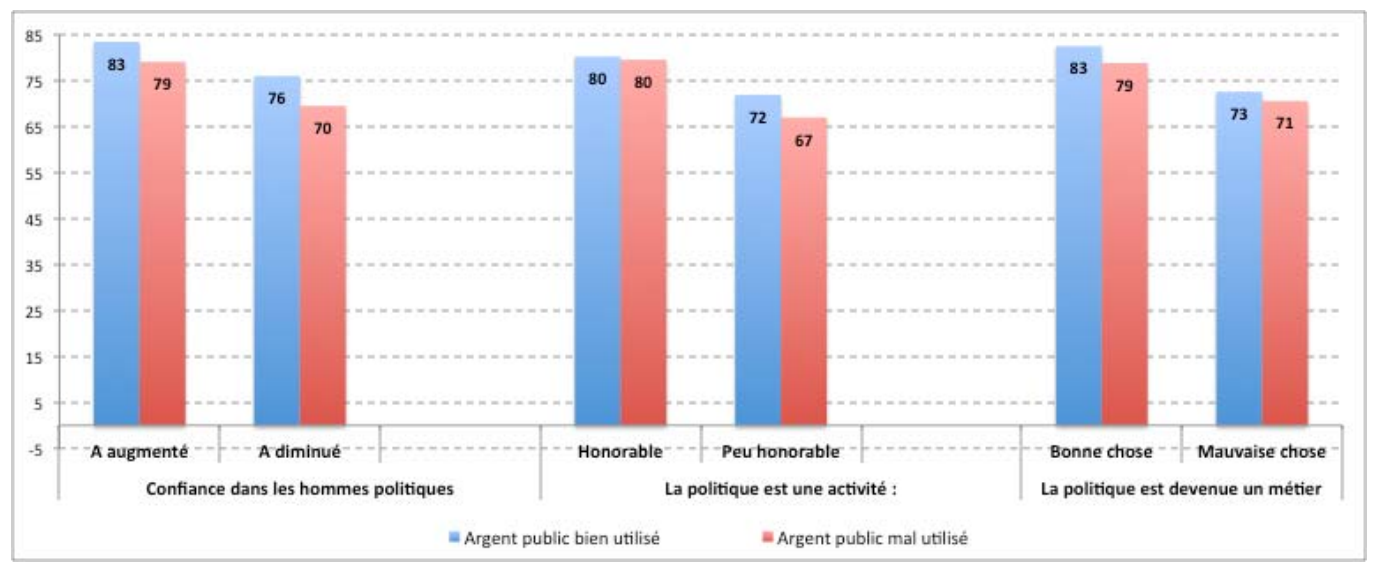

Figure $\mathrm{N}^{\circ}$ IV : Répartition des personnes ayant une image positive de l'argent selon leur opinion sur l'utilisation de l'argent public et leurs attitudes à l'égard du politique.

Les résultats pour cette troisième variable confirment les résultats précédents (figure nº IV). Le fait de considérer l'argent public mal utilisé est relié de façon substantielle à une image moins souvent positive de l'argent (de -1 à -6 points selon les situations)

Et de la même manière (figure ${ }^{\circ} \mathrm{V}$ ), si le fait d'exprimer une confiance forte vis-à-vis des acteurs politiques s'accompagne d'une perception positive de l'argent, celle-ci est moins manifeste si l'on considère l'argent public mal utilisé. Pour les personnes les plus confiantes à l'égard du politique (niveau 3) l'écart est de 7 points entre celles qui considèrent que l'argent public est bien utilisé ou qu'il l'est mal). 


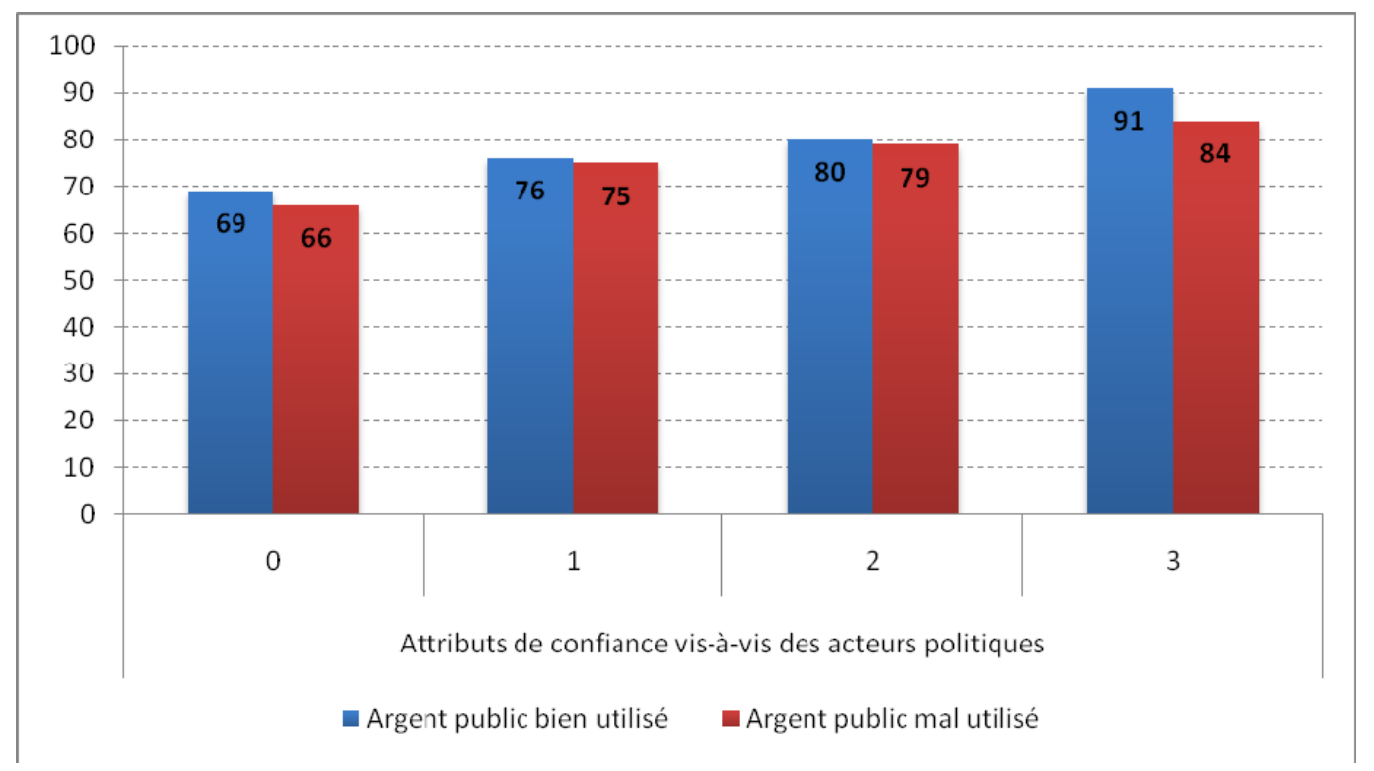

Figure $n^{\circ} \mathrm{V}$ : Répartition des personnes ayant une image positive de l'argent selon leur opinion sur l'utilisation de l'argent public et leur attribut de confiance à l'égard du politique

\section{< T1 > III - Conception de l'argent et jugement sur la probité publique}

Nous avons aussi exploré les relations existant entre les perceptions de l'argent et les jugements portés sur la probité des élus. Le croisement des variables confirme les résultats de la section précédente (II), à savoir un fort lien statistique entre l'image positive/négative de l'argent et les jugements portés sur le degré de corruption des acteurs publics (dirigeants politiques et institutions ${ }^{21}$ ). Cette dernière dimension est envisagée sous trois formes. Tout d'abord, un jugement sur l'honnêteté/la corruption des dirigeants. Dans l'enquête la répartition est la suivante : plutôt honnête 35\%, plutôt corrompu 60\% (NSP 5\%). Ensuite nous prendrons en compte l'appréciation de l'évolution de la corruption. Les réponses globales sont : plus qu'avant 33\%, autant 62\%, moins 4\% (NSP 1\%). Enfin, nous utiliserons une échelle mesurant l'attitude à l'égard du clientélisme et du favoritisme. Elle a été construite à partir des jugements d'acceptation ou de refus d'un certain nombre de comportements

\footnotetext{
${ }^{21}$ Nous utilisons ici deux questions de l'enquête. La première est posée sous cette forme depuis plus de vingt ans ce qui permet des comparaisons : « Diriez-vous qu’en règle générale, les élus et les dirigeants politiques français sont plutôt honnêtes ou plutôt corrompus ? ». Résultat global de l'enquête en 2008 : plutôt honnêtes 35\%, plutôt corrompus 60\%, NSP 5\% - La seconde est : « Pensez-vous qu'en France : il y a plus de corruption qu'avant ; il y en a autant mais on en parle davantage; il y en a plutôt moins ». Résultat global : plus de corruption qu'avant 33\% ; il y en a autant mais on en parle davantage 62\% ; il y en a plutôt moins, $4 \%$; NSP 1\%.
} 
montrant un citoyen à la recherche d'un passe-droit ${ }^{22}$. La population enquêtée se répartit ainsi : tolérance élevée du clientélisme 23 \%, tolérance moyenne 37 \%, réprobation 40 \%.

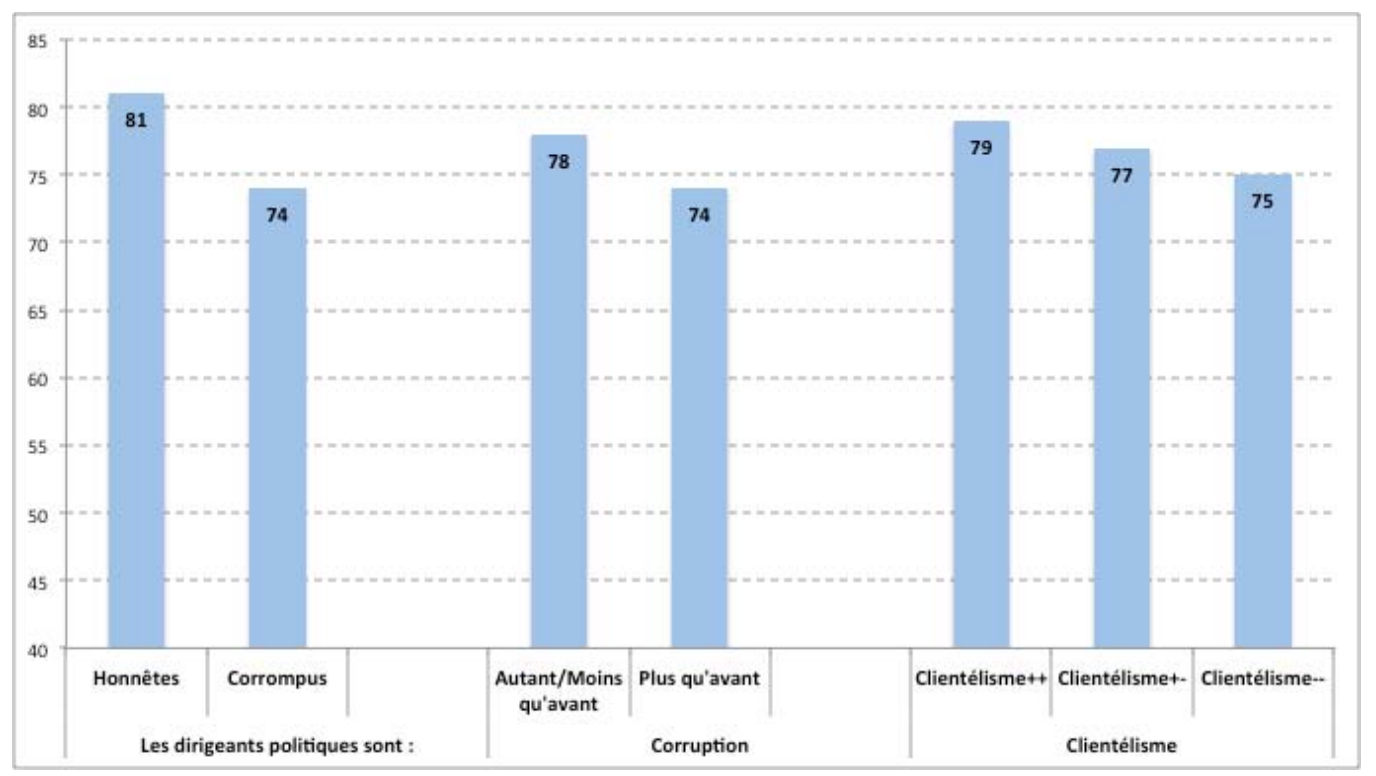

Fig $n^{\circ}$ VI - Jugements sur la probité des dirigeants en fonction de l’image de l’argent

La figure $\mathrm{n}^{\circ} \mathrm{VI}$ présente les jugements sur la probité en fonction de l'image positive/négative de l'argent. Globalement une image positive est reliée à une confiance dans la vie publique et à une tendance à la tolérance du clientélisme. Inversement une image négative de l'argent est associée à une plus grande défiance à l'égard des dirigeants et une réprobation plus forte du clientélisme. Reprenons chaque variable l'une après l'autre :

- 81\% des personnes estimant les élus honnêtes ont une image positive de l'argent (19\% ont une image négative) ; elles sont $74 \%$ quand elles estiment les élus corrompus, soit 7 points de moins ( $26 \%$ ont une image négative).

- 78\% des personnes estimant que la corruption n'a pas augmenté ont une image positive de l'argent (22\% ont une image négative) ; elles sont $74 \%$ quand elles considèrent que la corruption a augmenté (26\% ont une image négative).

- 79\% des personnes tolérant le clientélisme ont une image positive de l'argent (21\% ont une image négative) ; elles sont $75 \%$ quand elles réprouvent ces pratiques (25\% ont une image négative).

\footnotetext{
${ }^{22}$ Recommandation politique pour avoir une place en crèche, utilisation de relations politiques pour obtenir un emploi municipal, adhésion à un parti pour obtenir un logement social, acceptation d'un cadeau important par un client.
} 
Ce résultat dénote une conception dominante de la probité publique qui conjugue une confiance dans les dirigeants (plutôt honnêtes) et les institutions (peu corrompues) avec une acceptation des pratiques politiques « grises » et des arrangements.

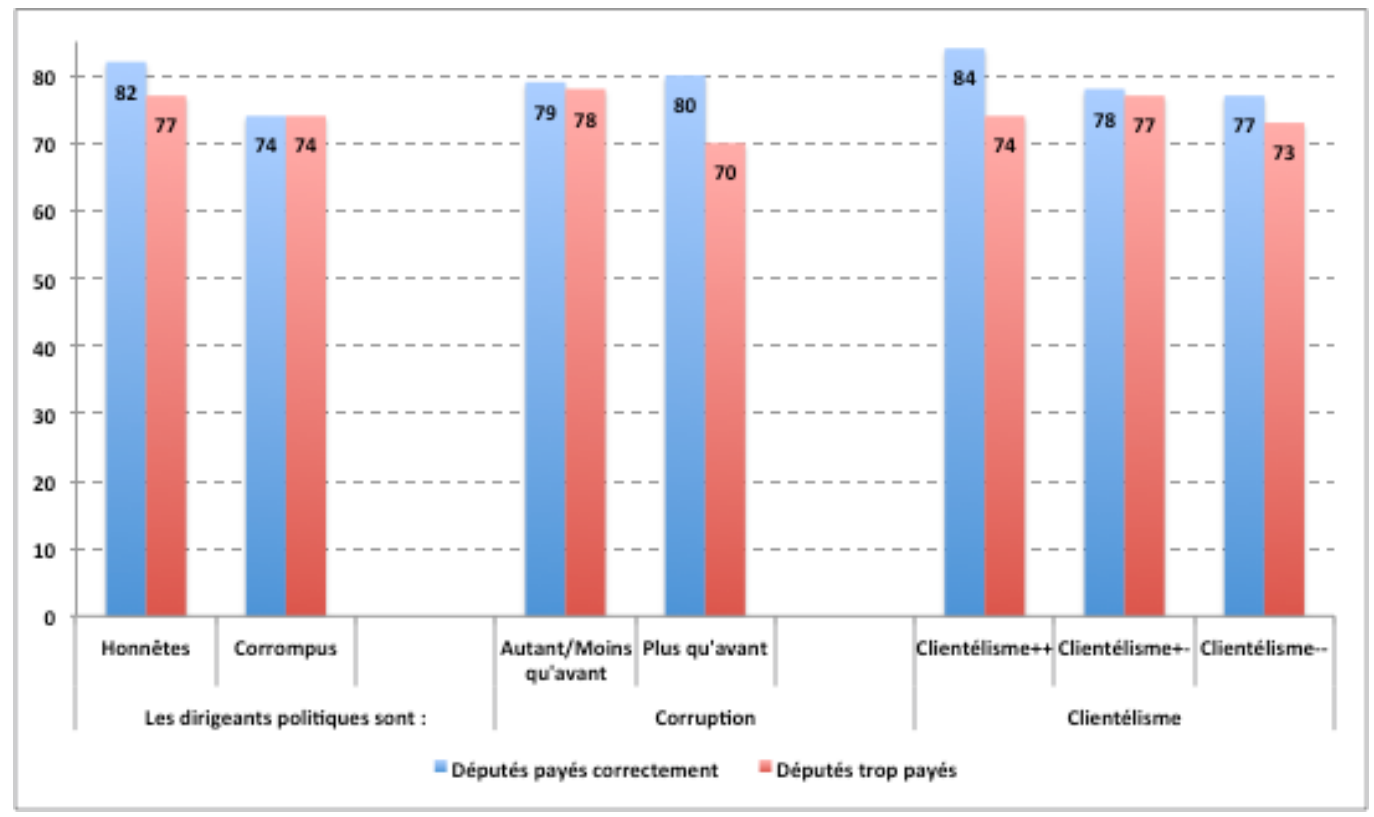

Fig $n^{\circ}$ VII Jugements sur la probité des dirigeants et sur leur rémunération en fonction de l'image de l'argent

La perception du niveau de rémunération des élus n'introduit pas de différences très marquées. 77\% des personnes estimant les élus honnêtes et les députés trop payés ont une image positive de l'argent (23\% ont une image négative). Elles sont $82 \%$ quand elles jugent les élus honnêtes et les députés payés correctement (18\% ont une image négative). Les différences sont plus affirmées quand il s'agit d'estimer l'évolution de la corruption (autant ou moins qu'avant/plus qu'avant). $70 \%$ des personnes estimant que la corruption augmente et que les députés sont trop payés ont une image positive de l'argent (30\% ont une image négative). Elles sont $79 \%$ à avoir une image positive de l'argent quand elles estiment la corruption stable et les députés payés correctement (21\% ont une image négative).

La position sur l'échelle de tolérance au clientélisme introduit aussi des écarts. 73\% des personnes défavorables au clientélisme et estimant les députés trop payés ont une image positive de l'argent (27\% ont une image négative). Elles sont $84 \%$ à avoir une image positive de l'argent quand elles sont tolérantes au favoritisme et estiment les députés payés correctement (16\% ont une image négative). 


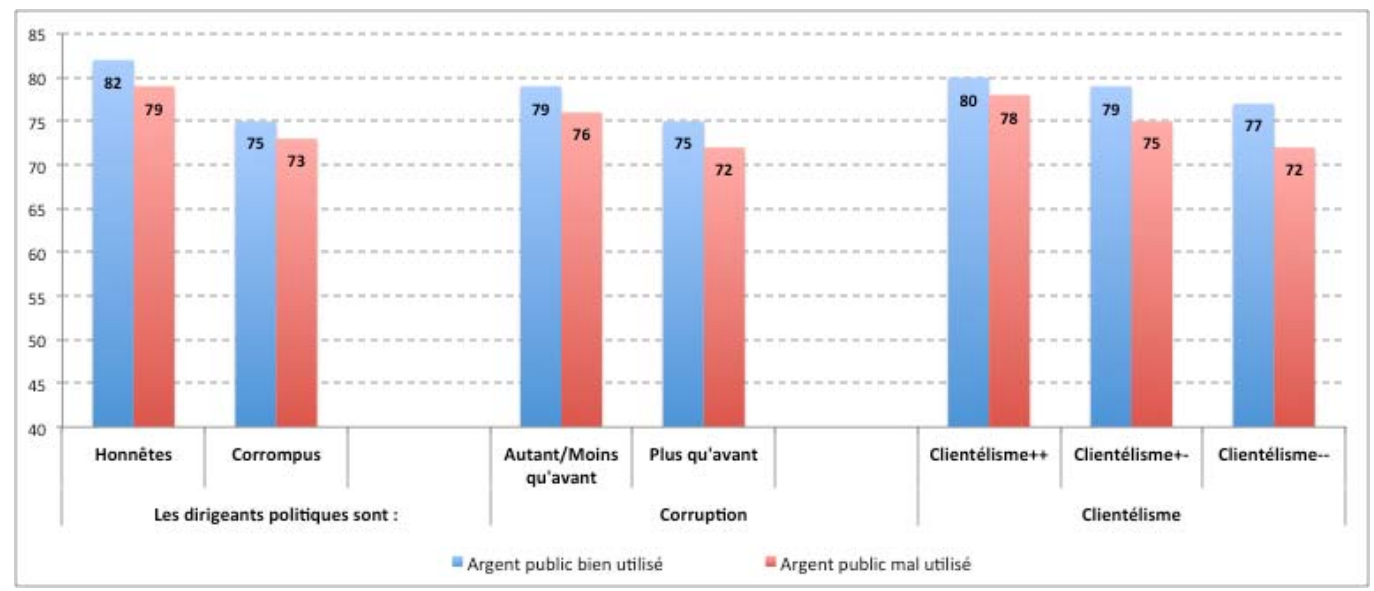

Fig $n^{\circ}$ VIII - Jugements sur la probité des dirigeants et sur l'utilisation des fonds public en fonction de l'image de l'argent

La perception de l'usage de l'argent public est une variable qui introduit des différenciations significatives dans la population enquêtée. Ainsi $82 \%$ des personnes qui estiment les élus honnêtes et l'argent public bien utilisé ont une image positive de l'argent (18\% ont une image négative). Elles ne sont plus que 73\% quand elles estiment les élus corrompus et l'argent public mal utilisé (27\% ont une image négative).

Les variations sont de même ampleur pour l'appréciation de l'évolution de la corruption. 79\% des personnes qui estiment le niveau de corruption stable et l'argent public bien utilisé ont une image positive de l'argent (21\% ont une image négative). Elles ne sont plus que $72 \%$ quand elles estiment qu'il y a aujourd'hui plus de corruption et que l'argent public mal utilisé (28\% ont une image négative).

L'attitude à l'égard du clientélisme a aussi des effets sensibles. $80 \%$ des personnes qui sont tolérantes au clientélisme et estiment que l'argent public est bien utilisé ont une image positive de l'argent ( $20 \%$ ont une image négative). Elles ne sont plus que $72 \%$ quand elles rejettent le clientélisme et considèrent l'argent public mal utilisé (28\% ont une image négative).

\section{$<$ IT1 $>$ IV - Espace de jugement de la relation entre Argent et politique}

Une analyse des correspondances multiples spécifique (ACM spécifique ${ }^{23}$ ) nous a permis de traiter l'ensemble des variables présentées jusqu'à présent et d'analyser leur combinaison. L'intérêt de ce type de méthode réside dans la possibilité de prendre en compte

\footnotetext{
${ }^{23}$ Sur la présentation de la méthode de l’ACM spécifique, voir l'annexe².
} 
simultanément tout un jeu de variables et d'en synthétiser les principales polarisations selon une réflexion multidimensionnelle.

- Les dix questions que nous avons placées en variables actives (qui structurent le champ délimité) sont celles relatives à l'image de l'argent et de l'enrichissement, au jugement sur la rémunération des députés et sur l'utilisation de l'argent public, ainsi que celles portant sur la perception de la fonction politique (confiance, honorabilité, professionnalisation) et la perception de la probité publique ${ }^{24}$;

- Plusieurs échelles d'attitude (relatives au favoritisme, à la confiance dans les institutions et les acteurs politiques, aux valeurs démocratiques), ainsi que des variables portant sur l'intérêt pour la politique et la participation électorale ont également été introduites dans l'analyse, en tant que variables complémentaires (ou variables illustratives). Nous avons aussi introduit à ce niveau les données socio-démographiques. Ces variables n'interviennent pas directement dans la structuration de l'espace et sont placées a posteriori dans l'espace des variables actives, afin de caractériser politiquement et socialement les principales polarisation observées).

Les résultats de cette ACM spécifique confirment l'importance des perceptions de l'argent dans les représentations sociales du "politique » (figure nº IX p. 27). En effet, l'espace des positions est organisé à partir de deux axes factoriels principaux qui permettent d'expliquer les grandes oppositions qui structurent les réponses à l'enquête.

- Le premier axe (axe horizontal sur la figure) concerne les attitudes à l'égard de la fonction politique (55\% de la variance du nuage $\mathrm{e}^{25}$ ): il oppose les attitudes d'adhésion (élus plutôt honnêtes, fonction honorable, professionnalisation bonne chose, députés payés correctement, argent public utilisé correctement, etc.) aux attitudes de défiance (élus plutôt malhonnêtes, fonction peu honorable, professionnalisation mauvaise chose, députés trop payés, argent public mal utilisé, etc.).

- Le deuxième axe (axe vertical sur la figure) est celui concernant la position vis-à-vis de l'argent (14\% de la variance du nuage) : il oppose l'attitude chrématiste (image positive de l'argent privé, jugement positif sur tous les moyens de l'obtenir, légitimes et illégitimes ${ }^{26}$ ) à celle anti-chrématiste (image négative de l'argent, jugement négatif sur tous les moyens de l’obtenir, illégitimes et légitimes).

\footnotetext{
${ }^{24}$ L’annexe 3 détaille les10 questions actives de l’ACM spécifique et précise les labels des modalités de réponses utilisées dans la figure IX

${ }^{25}$ Taux modifié (Benzécri J.P., 1992, Correspondence analysis handbook, New York, Dekker (adapté de Benzécri J.P., Benzécri F., 1984))

${ }^{26}$ bas de la figure $\mathrm{N}^{\circ} \mathrm{IX}$ p 27.
} 
- Un troisième axe, que nous n'utiliserons pas ici, intervient de façon secondaire et porte sur la définition des corrupteurs potentiels (5\% de la variance du nuage). Il oppose ceux qui mettent en cause les acteurs économiques (chefs d'entreprise, banques) à ceux qui mettent en cause le népotisme (la famille, les proches).

Sur cette base nous avons établi, par l'intermédiaire d'une classification ascendante hiérarchique, une typologie des positions individuelles. Les résultats confirment empiriquement l'importance de l'ambivalence dans les jugements portés sur les relations entre argent et politique (Runquist and al. 1977; Pharr, 1999). Cette complexité provient en particulier de la co-existence de jugements bien marqués (par rapport à nos deux axes principaux) et de jugements beaucoup plus ambigus. Ce résultat rejoint ceux qui ont été réalisés sur les perceptions des atteintes à la probité en politique et qui montrent que seul un peu plus de la moitié des personnes enquêtées (55\%) manifestent une attitude claire vis-à-vis de la corruption [qu'elle soit relativement tolérante (23\%) ou plus répressive (32\%)]. Les autres (45\%) mêlent forte réprobation et tolérance (27\%), ou minimisation de la corruption et forte réprobation (17\%) (Lascoumes and al. 2010).

Lorsque l'on combine les images vis-à-vis de l'argent privé avec celles à l'égard de la probité publique, il est possible de distinguer six groupes de position. Quatre d'entre eux, qui représentent $60 \%$ de notre population, sont bien caractérisés (tableau n5 et figure $n^{\circ} \mathrm{IX}$ ): les classes $n^{\circ} 2(23,5 \%, n=476), n^{\circ} 3(12,5 \%, n=253), n^{\circ} 4(12,3 \%, n=250)$, et $n^{\circ} 5(12 \%, n=$ 243).

Deux autres types sont plus hétérogènes la classe $n^{\circ} 1(20,3 \%, n=412)$ et la classe $n^{\circ} 6$ $(19,4 \%, n=394)$.

Nous pouvons synthétiser les résultats de la façon suivante par rapport aux deux axes principaux :

\begin{tabular}{|c|c|c|c|}
\hline \multirow{2}{*}{} & \multicolumn{2}{|c|}{ Axe 1 } \\
\cline { 3 - 4 } & & $\begin{array}{c}\text { Confiance dans les } \\
\text { institutions et les } \\
\text { acteurs politiques }\end{array}$ & $\begin{array}{c}\text { Défiance dans les } \\
\text { institutions et les } \\
\text { acteurs politiques }\end{array}$ \\
\hline \multirow{2}{*}{ Axe 2} & $\begin{array}{c}\text { Position anti- } \\
\text { chrématiste }\end{array}$ & Classe3 & $\begin{array}{c}\text { Classe 4 } \\
\text { Défiants non- }\end{array}$ \\
\hline & & $\begin{array}{c}\text { Confiants non- } \\
\text { matérialistes }\end{array}$ & $\begin{array}{c}\text { matérialistes } \\
12,3 \%, \mathrm{n}=250\end{array}$ \\
\hline
\end{tabular}




\begin{tabular}{|l|c|c|c|}
\hline & $\begin{array}{c}12,5 \%, \mathrm{n}=253 \\
\text { (qualifiés, âgés, } \\
\text { gauche) }\end{array}$ & $\begin{array}{c}\text { (peu qualifiés, } \\
\text { gauche) }\end{array}$ \\
\cline { 2 - 4 } & $\begin{array}{c}\text { Classe } \mathbf{2} \\
\text { Confiants } \\
\text { matérialistes } \\
\text { Position chrématiste } \\
\text { (qualifiés, droite) }\end{array}$ & $\begin{array}{c}\text { Classe 5 } \\
\text { Défiants matérialistes } \\
12 \%, \mathrm{n}=243 \\
\text { (peu qualifiés, jeunes } \\
\text { ni droite, ni gauche) }\end{array}$ \\
& $\begin{array}{c}\text { Proche }: \text { Classe 1 } \\
\text { (20,3\%, } n=412)\end{array}$ & $\begin{array}{c}\text { Proche }: \text { Classe 6 } \\
(19,4 \%, n=394)\end{array}$ \\
\hline
\end{tabular}

Tableau n5 - Typologie selon l’attitude vis à vis de l'argent privé et de la confiance politique

$\leq$ IT2 $>1$ - Deux Classes anti-chrématistes

- Les membres de la classe 3 sont anti-chrématistes. Ce sont des «confiants nonmatérialistes ». Ils ont une image plutôt négative de l'argent et ils sont très critiques sur les moyens illégitimes d'en gagner, mais aussi sur les moyens légitimes. Ils estiment que les députés sont plutôt trop payés.

C'est un groupe très confiant à l'égard des institutions et des acteurs politiques. Ils perçoivent la politique comme une fonction honorable, les élus comme plutôt honnêtes et le niveau de corruption stable. Ils considèrent aussi que l'argent public est plutôt bien utilisé. Enfin, ils s'intéressent à la politique, ils votent et s'estiment plutôt bien représentés.

Socialement c'est le groupe le plus âgé (plus de 67\% d'entre eux ont plus de 50 ans), dont les membres se retrouvent plutôt chez les retraités diplômés (cadres, ingénieurs, enseignants, professions intermédiaires), aux revenus plutôt élevés (19\% disposent de plus de 3000 euros mensuels). Ils se déclarent surtout de gauche et vivent souvent en milieu rural. Il y a parmi eux une importante proportion de catholiques pratiquants.

- Les membres de la classe 4 constituent le groupe le plus nettement anti-chrématiste, davantage que ceux la classe 3. Ce sont des «défiants non-matérialistes ». Ils ont une image très négative de l'argent et ils sont aussi critiques sur les moyens illégitimes de s'enrichir que sur les moyens légitimes.

Cependant, à l'inverse de la classe 3, ils partagent avec la classe 5 un ensemble de jugements critiques vis-à-vis du système politique. Ils sont défiants à l'égard des institutions et des acteurs politiques. Ils estiment que les députés sont trop payés et que l'argent public est plutôt mal utilisé. La politique est pour eux une fonction moyennement honorable. Ils considèrent 
que les élus sont plutôt corrompus et ils estiment qu'il y a aujourd'hui plus de corruption qu'avant. Ils sont défavorables à la professionnalisation. Ils s'intéressent peu à la politique et s'estiment mal représentés.

Socialement ce sont des personnes plutôt âgées, peu diplômées, aux revenus bas, ce sont surtout des employés, ouvriers et personnel de service. Ils se déclarent plutôt à gauche et vivent souvent dans de petites communes.

\section{<IT2> 2 - Deux classes chrématistes}

- Les membres de la classe 2 sont des «confiants matérialistes » Ils ont une image plutôt positive de l'argent, ils adhèrent fortement aux moyens légitimes d'en gagner et ils ne réprouvent que faiblement les moyens illégitimes. Ils estiment que les députés sont payés correctement (voire pas assez) et que l'argent public est plutôt bien utilisé.

C’est également le groupe le plus confiant vis-à-vis des institutions et des acteurs politiques. La politique est pour les membres de ce groupe une fonction honorable, les élus sont plutôt honnêtes et il n’y a pas aujourd'hui plus de corruption qu'avant. Ils s'intéressent à la politique, votent et s'estiment plutôt bien représentés.

Socialement, ce sont des personnes actives bien insérées, diplômées, des adultes et jeunes adultes vivant surtout dans de grandes villes. Ils se déclarent plutôt de droite. Il y a parmi eux une importante proportion de catholiques non pratiquants.

- Les membres de la classe 5 sont les plus nettement chrématistes. Ce sont des « défiants matérialistes » ils ont une image très positive de l'argent, ils adhèrent fortement à tous les moyens d'en gagner (légitimes et illégitimes).

Mais ils se distinguent nettement du groupe précédent par un ensemble de jugements critiques vis-à-vis des institutions et des acteurs politiques. Ils estiment que les députés sont trop payés et que l'argent public est plutôt mal utilisé.

Ils considèrent la politique comme une fonction moyennement honorable, les élus comme plutôt corrompus et ils estiment qu'il y a aujourd'hui plus de corruption qu'avant. Ils s’intéressent peu à la politique et s'estiment mal représentés.

Socialement ce sont des personnes peu ou pas diplômées (38\% ont au mieux un niveau primaire), plutôt jeunes (37,5\% ont moins de 35ans), ce sont surtout des employés, ouvriers et personnel de service. Ils se déclarent ni à droite ni à gauche ${ }^{27}$.

\footnotetext{
${ }^{27}$ Ce positionnement est souvent assimilé en France à une attitude favorable à l'égard de l'extrême droite
} (Mayer, 2002) 


\section{$\leq \mathrm{IT} 2>3$ - Deux types ambigus}

Deux classes sont en position intermédiaire par rapport aux précédentes. Et c’est précisément la question de leur position spécifique vis-à-vis de l'argent (attitude chrématiste/antichrématiste) qui les différencie:

- la classe 1 (20\%) est intermédiaire entre les classes 2 et 3. Elle regroupe des personnes au matérialisme modéré et confiantes. Elles sont moins anti-chrématistes que celles de la classe 3 (elles réprouvent, mais plus faiblement, le recours aux moyens légitimes et non légitimes). Elles partagent avec ce troisième groupe une confiance élevée à l'égard des institutions et des acteurs politiques.

Sociologiquement le groupe est très composite (avec tendanciellement des personnes plutôt qualifiées, des libéraux, des commerçants et artisans et des inactifs).

- la classe 6 (19\%) est intermédiaire entre les classes 4 et 5 . Elle regroupe des personnes au matérialisme normé et défiantes. Elles ont une position chrématiste moins affirmée que celles de la classe 5. Si elles partagent une image positive de l'argent et approuvent les moyens légitimes de s'enrichir, elles réprouvent les moyens non légitimes de le faire. Elles sont aussi très critiques à l'égard des institutions et des acteurs politiques (elles sont plus défiantes et portent des jugements plus sévères sur le politique que la classe 5).

Les membres de ce groupe sont jeunes, peu qualifiés, souvent de sexe féminin. 


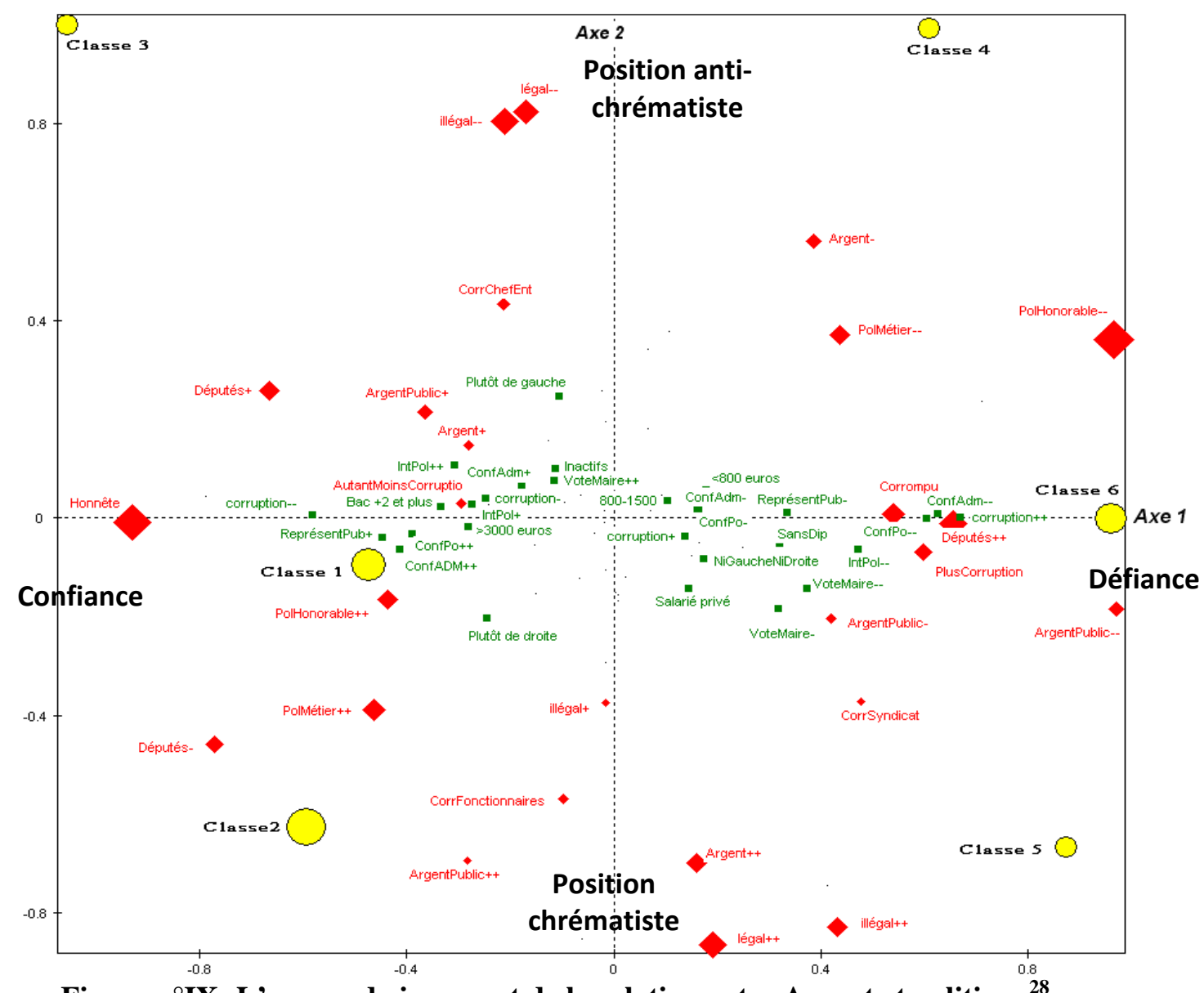

Figure $n^{\circ} I X-L^{\prime}$ 'espace de jugement de la relation entre Argent et politique ${ }^{28}$

${ }^{28}$ Modalités des variables actives (en rouge) et supplémentaires (en vert) qui contribuent au plan factoriel. 


\section{Conclusion}

Cette recherche permet de préciser sous un angle quantitatif les relations complexes et souvent antagonistes entre argent et politique. Trois conclusions principales peuvent être dégagées. Tout d'abord, nous avons montré l'existence d'une relation entre conception de l'argent privé et perception de l'argent dans les activités politiques. Nous avons établi une relation entre une image positive de l'argent et de l'enrichissement privé, et l'acceptation plus grande du rôle de l'argent dans les activités politiques. Inversement, une image négative de l'argent et de l'enrichissement privé s'accompagne d'une réprobation plus marquée du rôle de l'argent en politique.

Ensuite, nous avons mis en évidence la persistance de la dichotomie axiologique classique entre les attitudes chrématistes et anti-chrématistes. L'image ancestrale de «l'argent qui pourrit tout » reste d'actualité. Ce résultat conduit à réviser le postulat contemporain de l'impérialisme des valeurs économiques. Dans la société contemporaine, le statut social de l'argent et de l'enrichissement demeurent discuté par une partie non négligeable (un tiers) de la population enquêtée. Symétriquement, les chrématistes, les défenseurs absolus d’un tout économique (où la fin justifierait les moyens) sont minoritaires (22\%). Près de la moitié de notre échantillon a en ce domaine des positions mitigées sur la valeur sociale de l'argent et sur les moyens d'enrichissement.

Enfin, nous avons montré la force du clivage précédent dans sa combinaison avec les perceptions du politique et des enjeux de probité. Certes 39\% de la population enquêtée est difficile à positionner sur ces dimensions. C'est autant une limite de la méthode utilisée qu'un indicateur de complexité. Mais les 61\% restants constituent quatre classes bien différenciées qui sont illustratives du caractère multidimensionnel des perceptions de la " corruption » (des divers abus de fonction) et de ses causes (en particulier économiques et financières). A contrario, ces conclusions confirment la fragilité des sondages qui ne captent que des opinions de surface et des analyses qui dramatisent régulièrement les jugements en forme de «tous pourris » appliqués aux responsables politiques. 
Annexe 1 - Profils socio-économiques et rapport à l'argent et aux moyens de

\section{s'enrichir}

\begin{tabular}{|c|c|c|c|c|c|c|}
\hline & & $\begin{array}{c}\text { Image } \\
\text { positive } \\
\text { de } \\
\text { l'argent }\end{array}$ & $\begin{array}{c}3 \text { à } 4 \\
\text { moyens } \\
\text { légitimes } \\
\text { sur } 4 \\
\text { considérés } \\
\text { tout à fait } \\
\text { normaux }\end{array}$ & $\begin{array}{c}2 \text { à } 4 \\
\text { moyens } \\
\text { illégitimes } \\
\text { sur } 4 \\
\text { considérés } \\
\text { normaux }\end{array}$ & $\begin{array}{c}\text { Pro- } \\
\text { chrématistes }\end{array}$ & $\begin{array}{c}\text { Anti- } \\
\text { chrématistes }\end{array}$ \\
\hline \multirow{5}{*}{ 感 } & $18-24$ ans & 82 & 56 & 61 & 36 & 20 \\
\hline & 25-34 ans & 75 & 60 & 48 & 31 & 22 \\
\hline & $35-49$ ans & 75 & 56 & 39 & 23 & 28 \\
\hline & 50-64 ans & 78 & 50 & 27 & 16 & 40 \\
\hline & 65 ans et plus & 77 & 44 & 18 & 10 & 48 \\
\hline \multirow{4}{*}{ 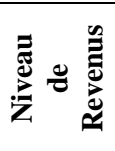 } & $<800 €$ & 71 & 50 & 36 & 20 & 35 \\
\hline & $800-1500 €$ & 75 & 51 & 34 & 21 & 36 \\
\hline & $1500-3000 €$ & 77 & 53 & 36 & 21 & 32 \\
\hline & $>3000 €$ & 82 & 53 & 37 & 25 & 35 \\
\hline \multirow{4}{*}{ 总总 } & Employeur, indépendant & 78 & 57 & 44 & 24 & 23 \\
\hline & $\begin{array}{l}\text { Salarié de l'Etat ou service } \\
\text { public }\end{array}$ & 71 & 53 & 38 & 24 & 33 \\
\hline & Salarié du privé & 77 & 57 & 42 & 26 & 27 \\
\hline & Inactif (dont retraité) & 78 & 49 & 28 & 17 & 40 \\
\hline \multirow{9}{*}{ 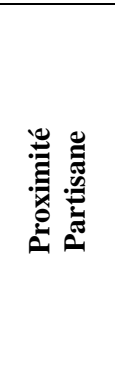 } & Parti d'extrême gauche* & 59 & 41 & 44 & 20 & 35 \\
\hline & PC & 71 & 44 & 27 & 21 & 50 \\
\hline & PS & 76 & 45 & 30 & 15 & 41 \\
\hline & Verts & 72 & 47 & 44 & 24 & 33 \\
\hline & $\begin{array}{l}\text { UDF (force démocrate } \\
\text { inclus) }\end{array}$ & 77 & 56 & 32 & 20 & 32 \\
\hline & UMP (RPR inclus) & 81 & 63 & 31 & 23 & 29 \\
\hline & $\mathrm{FN}$ & 84 & 60 & 44 & 28 & 24 \\
\hline & Autre* & 64 & 44 & 28 & 17 & 44 \\
\hline & Aucun parti & 79 & 57 & 40 & 25 & 28 \\
\hline \multirow{9}{*}{ 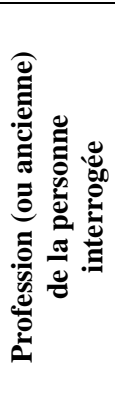 } & Agriculteur exploitant* & 81 & 55 & 31 & 22 & 36 \\
\hline & Artisan, commerçant & 83 & 59 & 36 & 23 & 28 \\
\hline & $\begin{array}{l}\text { Chef d'entreprise, prof. } \\
\text { Libérale* }\end{array}$ & 81 & 57 & 38 & 14 & 19 \\
\hline & Enseignant, Chercheur* & 76 & 33 & 21 & 12 & 59 \\
\hline & Cadre, ingénieur & 78 & 46 & 37 & 21 & 37 \\
\hline & Prof. Intermédiaire & 74 & 56 & 30 & 20 & 35 \\
\hline & Employé & 74 & 54 & 34 & 22 & 33 \\
\hline & $\begin{array}{l}\text { Ouvrier, personnel de } \\
\text { service }\end{array}$ & 77 & 52 & 38 & 22 & 32 \\
\hline & Inactif (autres que retraités) & 80 & 54 & 40 & 24 & 30 \\
\hline \multirow{5}{*}{ 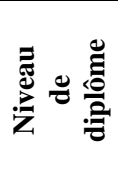 } & Sans diplôme & 78 & 51 & 36 & 21 & 34 \\
\hline & Primaire & 78 & 48 & 21 & 12 & 43 \\
\hline & Primaire sup. & 76 & 55 & 37 & 22 & 31 \\
\hline & Bac & 78 & 56 & 42 & 27 & 28 \\
\hline & $\mathrm{Bac}+2$ et plus & 76 & 53 & 40 & 25 & 32 \\
\hline \multirow{5}{*}{ 递 元 } & Cath. Pratiquants réguliers & 80 & 51 & 29 & 18 & 38 \\
\hline & Cath. Pratiquants irréguliers & 73 & 48 & 31 & 21 & 42 \\
\hline & Cath. Non pratiquants & 79 & 57 & 34 & 22 & 31 \\
\hline & Autres religions & 78 & 60 & 41 & 24 & 23 \\
\hline & Sans religion & 74 & 48 & 40 & 22 & 35 \\
\hline
\end{tabular}

* Effectifs faibles 
Annexe 2 - Qu’est-ce que l’analyse des correspondances multiples spécifique?

L'analyse des correspondances multiples (ACM) ${ }^{29}$ permet d'identifier les principales polarisations au sein d'une population. Ces clivages sont mesurés à partir de variables actives qui vont résumer les dimensions sous-jacentes au tableau de données analysées. Il s'agit d'une méthode géométrique, basée sur un calcul des distances entre individus ${ }^{30}$ (plus les réponses aux questions sélectionnées entre deux individus seront différentes, plus la distance sera importante). Les individus sont alors organisés selon un nuage de points (résumant ces distances), qui permet la détermination d'axes factoriels (axes principaux), hiérarchisés en fonction du degré de clivages qu'ils suscitent (du plus important au plus résiduel). Chaque individu obtient un score qui donne sa coordonnée factorielle sur chacun des axes. À partir de ces coordonnées factorielles individuelles, il devient possible de déterminer les points moyens des modalités de variables qui n’ont pas servi dans le calcul des distances entre individus. Nous les appelons classiquement variables illustratives ou supplémentaires. Il s'agit le plus souvent de variables sociodémographiques qui servent à « typer » les polarisations mises en évidence en amont (âge, sexe, diplôme, etc.). Les indicateurs politiques (vote aux élections, proximité partisane, positionnement politique, etc.) seront également utilisés de manière illustrative, afin de caractériser les clivages obtenus au sein des variables actives et d'en apporter des éléments d'explication.

L'ACM spécifiqueest une variante de l'ACM «classique » qui conserve les mêmes propriétés statistiques. Elle permet de classer des individus pour lesquels il manquerait certaines réponses à telle ou telle variable active, ou encore des individus qui choisiraient des modalités de réponse trop faiblement représentées dans l'échantillon ou des modalités de nonintérêt (catégorie "autre », etc.). Par cette méthode, ces individus sont conservés dans l'analyse, mais ponctuellement considérés comme "passifs » dans le calcul des distances entre individus. Cette méthode permet notamment d'annuler deux écueils. 1) Supprimer les individus caractérisés par des non-réponses ou des réponses rares (cela revient à travailler sur un échantillon tronqué, qui n’est donc plus représentatif de la population étudiée). 2) Conserver les modalités rares : cela conduit souvent à obtenir sur un des premiers axes factoriels l'opposition de ces individus « rares » aux autres.

\footnotetext{
${ }^{29}$ Pour davantage d'éclairages statistiques, se référer à Henry Rouanet et Brigitte Le Roux, Analyse des données multidimensionnelles. Statistique en sciences humaines, Paris, Dunod, 1993 ; Brigitte Le Roux et Henry Rouanet, "L'analyse multidimensionnelle des données structurées », Mathématiques et Sciences Humaines, 5 (18), 1984, p. 5-18.Pour un exemple empirique, se référer à Jean Chiche, Brigitte Le Roux, Pascal Perrineau et Henry Rouanet, "L'espace politique des électeurs français à la fin des années 1990. Nouveaux et anciens clivages, hétérogénéité des électorats », Revue française de science politique, 50 (3), juin 2000, p. 463-487.

${ }_{30}$ «Individu » est entendu ici au sens statistique du terme.
} 


\section{Annexe 3 - Les 10 variables actives de l'analyse des correspondances multiples spécifique}

Q1- Je vais vous citer une liste de mots. Pour chacun d'eux, vous me direz s'ils évoquent pour vous quelque chose de positif ou de négatif : Argent

$\begin{array}{ll}\text { - Très positif (Argent }++ \text { ) } & 29 \% \\ \text { - Assez positif (Argent }+ \text { ) } & 48 \% \\ \text { - Plutôt, très négatif (Argent-) } & 23 \% \\ \text { - NSP (ArgentNSP) } *^{31} & 0,3 \%\end{array}$

Q2- . D'une manière générale, diriez-vous que les députés sont... ?

- Trop payés (Députés++) 50\%

- Assez bien payés (Députés+) 29\%

- Payés juste comme il faut / pas assez payés (Députés-) 15\%

- NSP (DéputésNSP) * 6\%

Q3- Pensez-vous qu'en France ...

- Il y a plus de corruption qu'avant (PlusCorruption) 33\%

- Il y en a autant, mais on en parle davantage / Il y en a plutôt moins

(AutantMoinsCorruption)

- NSP (CorruptionNSP)

Q4- Quand un élu est accusé de corruption, d'autres personnes sont souvent complices. Lesquelles selon vous ... : en premier

- Ses électeurs / Des membres de sa famille (CorrElecteursFamille) 21\%

- Des chefs d'entreprises (CorrChefEnt) 33\%

- Des fonctionnaires (CorrFonctionnaires) 16\%

- Des responsables syndicaux (CorrSyndicat) $\quad 7 \%$

- Des dirigeants de banques (CorrBanques) $\quad 16 \%$

- Aucune (CorrAucun) * 2\%

- NSP (CorrNSP) * $\quad 4 \%$

Q5- De manière générale, diriez-vous que l'argent public utilisé par les élus de votre commune est...

- Très bien utilisé (ArgentPublic++) 7\%

- Plutôt bien utilisé (ArgentPublic+) 54\%

- Plutôt mal utilisé (ArgentPublic-) 28\%

- Très mal utilisé (ArgentPublic--) 10\%

- NSP (ArgentPublicNSP) * 1\%

Q6- Diriez-vous que la politique est une activité ... ?

- Honorable (PolHonorable++) 67\%

- Peu honorable (PolHonorable--) 30\%

- NSP (PolHonorableNSP) * 3\%

31* signifie que la modalité a été placée en modalité passive dans l’analyse (cf. annexe 2). 
Q7- Diriez-vous qu'en règle générale les élus et les dirigeants politiques français sont...?

- Plutôt honnêtes (Honnête) 35\%

- Plutôt corrompus (Corrompu) $\quad 60 \%$

- NSP (HonnCorrNSP) * 5\%

Q8- On dit aujourd'hui que la politique est devenue un métier. Diriez-vous que c'est... ?

- Plutôt une bonne chose (PolMétier++) 47\%

- Plutôt une mauvaise chose (PolMétier--) $\quad 48 \%$

- NSP (PolMétierNSP) * 5\%

Q9- nombre de moyens légitimes de gagner de l’argent considérés comme normaux

( " hériter », " épargner », " créer son entreprise », " faire des heures supplémentaires »)
- 0-1 moyen (légal--)
$28 \%$
- 2 moyens (légal-)
$19 \%$
- 3 moyens (légal+)
$26 \%$
-4 moyens (légal ++ )
$27 \%$

Q10- nombre de moyens illégitimes de gagner de l’argent considérés comme normaux

( « frauder le fisc », « cumuler des emplois », « employer des gens au noir », « utiliser des combines »)
- 0 moyen (illégal--)
$30 \%$
- 1 moyen (illégal-)
$34 \%$
- 2 moyens (illégal + )
$19 \%$
- 3-4 moyens (illégal++)
$17 \%$ 


\section{Bibliographie}

Algan Y., Cahuc P., 2007, La société de défiance, comment le modèle français s’auto-détruit, Paris, Editions rue d'Ulm.

Algan Y., Cahuc P., Zylbergerg A, La fabrique de la défiance, Paris, A. Michel, 2012

Bacot P., 1999, «En haut et du mauvais côté. Les professionnels de la politique au miroir des citoyens » in M. Offerlé, La profession politique. XIXe-XXe siècles, Paris, Belin, p. 307-331.

Bayley, D. H., 1966, “The effects of corruption in a developing nation », Western Poltical Quaterly, 19, 4, p. 719-732;

Briquet J.L..,Sawicki F., Le clientélisme politique dans les sociétés contemporaines, Paris, PUF, 1998.

Briquet J.L. et Garraud Ph. (dir.), 2001, Juger la politique. Entreprises et entrepreneurs critiques de la politique, Rennes, Presses Universitaires de Rennes,

Damamme, D., 1999, « Professionnel de la politique, un métier peu avouable », in Offerlé (M.), dir., La profession politique XIXe-XXe siècles, Paris, Belin, p. 37-67.

De Blic D., 2005, « Moraliser l’argent, ce que Panama a changé dans la société française », Politix, n'71, p. 61- 82

Della Porta D., Mény Y., 1995, Démocratie et corruption en Europe, Paris, La Découverte,

Desanti O., 1968, La banquière des années folles, Marthe Hanau, Fayard

Doganis C. ,2007, Aux origines de la corruption, démocratie et délation en Grèce ancienne, Paris, PUF.

Ferry J., 1868, Les comptes fantastiques d'Haussmann, Paris, Le Temps

Garrigues J., 2004, Les scandales de la République, Paris R. Laffont

Gaxie D. 2001, "Enchantements, désenchantements, réenchantements, les critiques profanes de la politique”, in Briquet J.L. et Garraud Ph., 2001, op cit

Heidenheimer, A, \& M. Johnston, LeVine, Th. V., 1989, Political Corruption: a Handbook, New Brunswick, N.J: Transaction,

Jeanneney, J.N., 1976, François de Wendel en république, Paris, Seuil

Jeanneney, J.N., 1981, L'argent caché, milieux d'affaires et pouvoir politique, Paris Fayard

Lambsdorff J. G., 2007, The institutional economics of corruption and reform, Cambridge, Cambridge

University Press.

Lascoumes P., 1997, Élites irrégulières : essai sur la délinquance d’affaires, « Scandale, affaire et problème », chap II, Paris, Gallimard, p.81-112.

Lascoumes P. (dir.), 2010, Favoritisme et corruption à la française, petits arrangements avec la probité, Paris, Presses de sciences-Po, p. 73-106.

Lascoumes P., 2011, Une démocratie corruptible, arrangements, favoritisme et conflits d'intérêts, Paris, Seuil.

Mayer, N., 2002 a , « Les dimensions de la confiance », La démocratie à l'épreuve, Presses de Sc-Po, p.88 s..

Mayer, N., 2002 b, Ces français qui votent Le Pen, Paris, Fayard

Médard J.F., "Le rapport de clientèle : du phénomène social à l'analyse politique”, Revue française de science politique, 1976, 26, 1, p. 103-131

Mélèze, J., 1987, « Sycophantes et délateurs, un mal dans la cité », La délation, Autrement, p. 35 s.

Mény, Y., 1992, La corruption de la République, Paris, Fayard.

Mollier J. Y., Le scandale de Panama, Paris Fayard, 1991

Neild, R., 2002, Public corrupion, the dark side of social evolution, London Althem Press, 2002,

Norris P. , 1999, Critical citizens: global support for democratic government, Oxford, Oxford University Press.

Orléan A., 2011, L'empire de la valeur, Paris, Seuil.

Padioleau, J.G., 1975, « De la corruption dans les oligarchies pluralistes », Revue française de sociologie, XVI, pp.33-58.

Pharr, S., Putnam, R.D., 2000, Disaffected democracies: what's troubling the trilateral countries?, Princeton, N.J. : Princeton University Press.

Phélippeau E., 2002, L'invention de l'homme politique moderne, Paris, Belin, 2002 
Pharr S. J., 1999, «Are Citizens Lax or Cynical? Corruption Tolerance and One-Party Dominance », European University Institute, Robert Schuman Centre, Conference on Political Corruption and Parties, March.

Pharr S., Robert D. Putnam, R., 2000, Disaffected democracies: what's troubling the trilateral countries?, Princeton, Princeton University Press, 2000

Piff P. K., Stancato D.M., and al., 2012, "Higher social class predicts increased unethical behavior”, Proccedings of National Academy of Sciences, PNAS Early Edition , 28 février.

Rivière Y., 2005, «Rome impériale: les délateurs le prince, le tribunal », Citoyens et délateurs, Autrement, $\mathrm{n}^{\circ} 238$, p. $26 \mathrm{~s}$.

Rosanvallon P., 2006, La Contre-démocratie : la politique à l'âge de la défiance, Paris Seuil, p. 19-38

Runquist B. S., Strom G.S., Peters J.G.,1977, « Corrupt Politicians and Their Electoral Support : Some

Experimental Observations », American Political Science Review, 3, 1977, p. 956-974.

Simmel G. (1900) 1987, Philosophie de l'argent, Paris, PUF

Sombart W. , 1902, Der moderne Kapitalismus, Munich, Duncker und Humblot.

Steffens L., 1904, The shame of the cities, New York, P. Smith

Weber M. (1904), 2003, Ethique protestante et esprit du capitalisme, Paris, Gallimard. 